\title{
Eventual periodicity for the KdV equation on a half-line
}

\author{
Jie Shen $^{\mathrm{a}}$, Jiahong $\mathrm{Wu}^{\mathrm{b}, *}$, Juan-Ming Yuan ${ }^{\mathrm{c}}$ \\ ${ }^{a}$ Department of Mathematics, Purdue University, West Lafayette, IN 47907, USA \\ ${ }^{\mathrm{b}}$ Department of Mathematics, Oklahoma State University, 401 Mathematical Sciences, Stillwater, OK 74078, USA \\ ${ }^{\mathrm{c}}$ Department of Applied Mathematics, Providence University, 200 Chung-Chi Road, Shalu 433, Taichung Hsien, Taiwan \\ Received 18 July 2006; received in revised form 2 February 2007; accepted 9 February 2007 \\ Available online 16 February 2007 \\ Communicated by B. Sandstede
}

\begin{abstract}
This paper studies the eventual periodicity of solutions to the initial and boundary value problem for the KdV equation on a half-line and with periodic boundary data. We derive a representation formula for solutions to the linearized KdV equation and rigorously establish the eventual periodicity of these solutions. Numerical experiments performed on the full KdV equation indicate that its solutions are also eventually periodic. (C) 2007 Elsevier B.V. All rights reserved.
\end{abstract}

Keywords: Eventual periodicity; Linearized KdV equation; KdV equation; Numerical solution

\section{Introduction}

This paper is concerned with the eventual periodicity of solutions to the initial- and boundary-value problem (IBVP) for the $\mathrm{KdV}$ equation on the half-line:

$$
\begin{cases}u_{t}+u_{x}+u u_{x}+u_{x x x}=0, & x \geq 0, t \geq 0 \\ u(x, 0)=u_{0}(x), & x \geq 0, \\ u(0, t)=g(t), & t \geq 0\end{cases}
$$

Assuming the boundary data $g$ is a periodic function of period $T>0$, we investigate whether the corresponding solution $u$ of (1.1) is eventually periodic, in the sense that

$\lim _{t \rightarrow \infty}(u(x, t+T)-u(x, t))=0$

for any $x>0$.

This study was partially motivated by an observation of experiments involving surface water waves generated by a wavemaker mounted at one end of a water channel [1]. When the wavemaker oscillates periodically with a period $T$, it

\footnotetext{
* Corresponding author. Tel.: +1 405744 5788; fax: +1 4057448275 .

E-mail addresses: shen@math.purdue.edu (J. Shen), jiahong@math.okstate.edu (J. Wu), jmyuan@pu.edu.tw (J.-M. Yuan).
}

appears that the wave amplitude at each point down the channel becomes periodic after a certain amount of time. Our goal is to establish this experimental phenomenon as a mathematically rigorous fact for the IBVP (1.1).

We first point out that the eventual periodicity concerned here is not a trivial property. In contrast to the pure initialvalue problem for the $\mathrm{KdV}$ equation on the whole line, solutions to the IBVP (1.1) do not decay in time. In fact, when $g$ is periodic of period $T$, the $L^{2}$-norm of the corresponding solution to (1.1) grows at the order of $\sqrt{t}$ (see [3]). It remains an open problem whether the $L^{\infty}$-norm of the solution is bounded for all time. To deal with this difficulty, Bona, Sun and Zhang studied in [2] the eventual periodicity of an equation obtained by appending the damping term $u$ to the KdV equation. Assuming the boundary data is not too large, they were able to establish the eventual periodicity for this equation. In the work of Bona and $\mathrm{Wu}$ [3], the large-time behaviour of the $\mathrm{KdV}$ equation, the BBM equation and their counterparts with Burgers-type dissipation were comprehensively investigated. In particular, the eventual periodicity for solutions to the linearized version of these equations were established.

Our investigation on the eventual periodicity problem of (1.1) is carried out in two stages. In the first stage, we focus on the IBVP for the linearized $\mathrm{KdV}$ equation, namely 
$\begin{cases}u_{t}+u_{x}+u_{x x x}=f, & x \geq 0, t \geq 0, \\ u(x, 0)=u_{0}(x), & x \geq 0, \\ u(0, t)=g(t), & t \geq 0 .\end{cases}$

For each $x>0$ and $t \in[0, T]$, we consider the sequence $\{u(x, t+n T)\}_{1}^{\infty}$ and study its limit

$\lim _{n \rightarrow \infty} u(x, t+n T)$.

This limit, if it exists, would specify the time-dependent equilibrium that is reached in the channel. The eventual periodicity then follows as a special consequence. To compute this limit, we first derive the following representation formula for solutions of (1.2):

$$
\begin{aligned}
u(x, t)= & \int_{0}^{\infty} \Gamma(x, y, t) u_{0}(y) \mathrm{d} y \\
& +\int_{0}^{t} \int_{0}^{\infty} \Gamma(x, y, t-\tau) f(y, \tau) \mathrm{d} y \mathrm{~d} \tau \\
& +\int_{0}^{t} \Phi(x, t-\tau) g(\tau) \mathrm{d} \tau,
\end{aligned}
$$

where $\Gamma$ and $\Phi$ are two explicit kernel functions. The approach to deriving this formula is different from that in [3]. Instead of taking the Laplace transform with respect to $t$, we follow the idea of Hayashi, Kaikina and Guardado Zavala [5] by performing the Laplace transform with respect to $x$. We note that the authors of [5] focused on the $\mathrm{KdV}$ equation without the convection term $u_{x}$ and that the process is more complex when $u_{x}$ is present. We then analyse the large-time behaviour of the kernel functions $\Gamma$ and $\Phi$ and establish suitable asymptotic estimates through the theory of stationary phase. These estimates allow us to prove the existence of the limiting function in (1.3), which is periodic of period $T$ and assumes the boundary data $g$ at $x=0$. As a special consequence, we conclude the eventual periodicity of the linearized $\mathrm{KdV}$ equation.

We remark that the study of the linearized $\mathrm{KdV}$ equation is our first step towards a complete theory on the eventual periodicity of water waves. The results presented in this paper will be useful in the investigation of the eventual periodicity of the full $\mathrm{KdV}$ equation. In fact, our idea to deal with the full $\mathrm{KdV}$ equation is to use the representation formula here to recast the equation in an integral form and to apply the contraction mapping principle on a suitable functional setting such as a Banach space of functions satisfying the eventual periodicity.

At the second stage, we investigate the eventual periodicity of (1.1) through numerical experiments. We numerically compute the solutions of (1.1) corresponding to suitably chosen data and then plot these solutions and analyse their largetime behaviour. When we do the actual computations, we approximate this half-line problem by a two-point boundary value problem on the interval $[0, L]$ for sufficiently large $L$. We then scale the problem from $[0, L]$ to $[-1,1]$ and compute the solutions of the resulting problem using the Dual Petrov-Galerkin method proposed by Shen [8]. The numerical experiments indicate that the solutions of (1.1) corresponding to the selected boundary data are eventually periodic. We also computed solutions of the linearized $\mathrm{KdV}$ equation for the purpose of verifying our theory in the first stage. In addition, we included two dissipative versions of the $\mathrm{KdV}$ equation in our numerical study: the KdV-Burger equation (the $\mathrm{KdV}$ equation with the damping term $u_{x x}$ ) and the equation with the damping term $u$. The numerical results of these equations imply that these terms significantly damp the amplitudes of the solutions but maintain the pattern of eventual periodicity.

The rest of this paper is organized as follows. Section 2 is devoted to the theoretical study on the eventual periodicity of the linearized KdV equation. Section 3 contains the results of the numerical experiments on the eventual periodicity of the full $\mathrm{KdV}$ equation. Some background materials on the Laplace transform and some detailed asymptotic analysis on oscillatory integrals are provided in the Appendices.

\section{The linearized $\mathrm{KdV}$ equation}

This section is concerned with the eventual periodicity of the IBVP for the linearized KdV equation:

$\begin{cases}u_{t}+u_{x}+u_{x x x}=f, & x \geq 0, t \geq 0, \\ u(x, 0)=u_{0}(x), & x \geq 0, \\ u(0, t)=g(t), & t \geq 0 .\end{cases}$

We start our investigation by deriving a solution formula for this problem. It involves convolutions of two kernel functions $\Gamma(x, y, t)$ and $\Phi(x, t)$ with the data $u_{0}$ and $g$. We then establish the large-time asymptotics of $\Gamma$ and $\Phi$. As a consequence, we prove the convergence of any solution of (2.1) to a timedependent periodic equilibrium, which implies, in particular, the eventual periodicity. We end this section by mentioning the solution representation formula for the IBVP of the linearized $\mathrm{KdV}$-Burgers equation.

We start by fixing several notations. Let $\eta$ be a complex number with the real part $\operatorname{Re}(\eta)>0$. The cubic equation

$\xi^{3}+\xi+\eta=0$

always has two roots $\xi_{1}$ and $\xi_{2}$ with $\operatorname{Re}\left(\xi_{1}\right) \geq 0$ and $\operatorname{Re}\left(\xi_{2}\right) \geq 0$, and a third root $\xi_{3}$ with $\operatorname{Re}\left(\xi_{3}\right)<0$.

We use extensively the Laplace transform and the inverse Laplace transform. For a piecewise continuous function $F=$ $F(y)$ on $y \geq 0$, the Laplace transform of $F$ is defined by

$(\mathcal{L} F)(\xi)=\widehat{F}(\xi)=\int_{0}^{\infty} \mathrm{e}^{-\xi y} F(y) \mathrm{d} y$.

If the transform $\widehat{F}(\xi)$ is known, then $F(y)$ is called the inverse Laplace transform of $\widehat{F}(\xi)$ and we write

$F=\mathcal{L}^{-1} \widehat{F}$.

The general inverse formula is given by

$F(y)=\left(\mathcal{L}^{-1} \widehat{F}\right)(y)=\frac{1}{2 \pi \mathrm{i}} \int_{a-\mathrm{i} \infty}^{a+\mathrm{i} \infty} \mathrm{e}^{y \xi} \widehat{F}(\xi) \mathrm{d} \xi$

The integral here is a complex contour integral taken over the infinite straight line (called a Bromwich path) in the complex plane from $a-\mathrm{i} \infty$ to $a+\mathrm{i} \infty$. The number $a$ is any real 
number for which the resulting Bromwich path lies to the right of any singularities of $\widehat{F}(\xi)$. More details on the inverse Laplace transform can be found in the book [7]. For notational convenience, we will omit $a$ but still adopt this convention. We now state the major results of this section.

Theorem 2.1. Assume that

$u_{0} \in L^{1}(0, \infty), \quad g \in L^{\infty}(0, \infty)$ and

$f \in L^{\infty}\left(0, \infty ; L^{1}(0, \infty)\right)$.

Then the solution $u$ of the IBVP (2.1) can be written as:

$$
\begin{aligned}
u(x, t)= & \int_{0}^{\infty} \Gamma(x, y, t) u_{0}(y) \mathrm{d} y \\
& +\int_{0}^{t} \int_{0}^{\infty} \Gamma(x, y, t-\tau) f(y, \tau) \mathrm{d} y \mathrm{~d} \tau \\
& +\int_{0}^{t} \Phi(x, t-\tau) g(\tau) \mathrm{d} \tau
\end{aligned}
$$

where the kernel functions $\Gamma$ and $\Phi$ are given by

$$
\begin{aligned}
\Gamma(x, y, t)= & \frac{1}{2 \pi \mathrm{i}} \int_{-\mathrm{i} \infty}^{\mathrm{i} \infty} \mathrm{e}^{-\left(\xi^{3}+\xi\right) t} \mathrm{e}^{\xi(x-y)} \mathrm{d} \xi \\
& -\frac{1}{2 \pi \mathrm{i}} \int_{-\mathrm{i} \infty}^{\mathrm{i} \infty} \mathrm{e}^{\eta t} \mathrm{e}^{\xi_{3} x}\left(\mathrm{e}^{-\xi_{1} y} \xi_{1}^{\prime}+\mathrm{e}^{-\xi_{2} y} \xi_{2}^{\prime}\right) \mathrm{d} \eta \\
\Phi(x, t)= & \frac{1}{2 \pi \mathrm{i}} \int_{-\mathrm{i} \infty}^{\mathrm{i} \infty} \mathrm{e}^{-\left(\xi^{3}+\xi\right) t} \mathrm{e}^{\xi x}\left(\xi^{2}+1\right) \mathrm{d} \xi \\
& -\frac{1}{2 \pi \mathrm{i}} \int_{-\mathrm{i} \infty}^{\mathrm{i} \infty} \mathrm{e}^{\eta t} \mathrm{e}^{\xi_{3} x}\left(2 \xi_{3}^{2} \xi_{3}^{\prime}\right) \mathrm{d} \eta .
\end{aligned}
$$

Corollary 2.2. The kernel functions $\Gamma$ and $\Phi$ can be written in the following more compact form:

$$
\begin{gathered}
\Gamma(x, y, t)=-\frac{1}{2 \pi \mathrm{i}} \int_{-\mathrm{i} \infty}^{\mathrm{i} \infty} \mathrm{e}^{\eta t} \mathrm{e}^{\xi_{3} x}\left(\mathrm{e}^{-\xi_{1} y} \xi_{1}^{\prime}\right. \\
\left.+\mathrm{e}^{-\xi_{2} y} \xi_{2}^{\prime}+\mathrm{e}^{-\xi_{3} y} \xi_{3}^{\prime}\right) \mathrm{d} \eta, \\
\Phi(x, t)=\frac{1}{2 \pi \mathrm{i}} \int_{-\mathrm{i} \infty}^{\mathrm{i} \infty} \mathrm{e}^{-\left(\xi^{3}+\xi\right) t} \mathrm{e}^{\xi x}\left(3 \xi^{2}+1\right) \mathrm{d} \xi .
\end{gathered}
$$

Theorem 2.3. Let $\Gamma$ and $\Phi$ be defined as in (2.5) and (2.6):

(1) There exists a constant $C$ such that

$$
|\Gamma(x, y, t)| \leq \frac{C}{t^{1 / 2}}
$$

uniformly for $x, y \in[0, \infty)$ and for sufficiently large $t$;

(2) For any compact set $\left[A_{1}, A_{2}\right]$ with $0<A_{1}<A_{2}<\infty$, there exists a constant $C=C\left(A_{1}, A_{2}\right)$ such that

$$
|\Phi(x, t)| \leq \frac{C}{t^{3 / 2}}
$$

uniformly for $x \in\left[A_{1}, A_{2}\right]$ and for sufficiently large $t$.

The results of the above theorems allow us to show that any solution of the IBVP (2.1) with a periodic boundary datum $g$ converges to a time-dependent equilibrium periodic function when $f$ is identically zero.
Theorem 2.4. Let $f \equiv 0, u_{0} \in L^{1}(0, \infty)$ and $g \in C^{1}(0, \infty)$. Assume $g$ is periodic of period $T>0$. Let $u$ be the corresponding solution of the IBVP (2.1). Then, for any $x>0$ and $t>0$, the limit

$\lim _{n \rightarrow \infty} u(x, n T+t)=u_{\infty}(x, t)$

exists for some function $u_{\infty}$ that is periodic of period $T$ and satisfies

$u_{\infty}(0, t)=g(t)$.

As a special consequence, $u$ is eventually periodic in the sense that

$\lim _{t \rightarrow \infty}(u(x, T+t)-u(x, t))=0$

for any $x>0$ and uniformly for $x \in\left[A_{1}, A_{2}\right]$ with $0<A_{1}<$ $A_{2}<\infty$.

Remark. As we shall see in the proof of this theorem, the initial datum $u_{0}$ does not contribute to the time-dependent equilibrium $u_{\infty}$.

For the clarity of presentation, we divide the rest of this section into three subsections.

\subsection{Solution representation}

This subsection derives the solution representation formula given in Theorem 2.1.

Proof of Theorem 2.1. We denote the Laplace transform of $u(x, t)$ with respect to $x$ by $\widehat{u}(\xi, t)$, namely

$\widehat{u}(\xi, t)=\int_{0}^{\infty} \mathrm{e}^{-x \xi} u(x, t) \mathrm{d} x$.

Taking the Laplace transform of the linear $\mathrm{KdV}$ equation with respect to $x$ and applying Proposition A.2 of Appendix A, we obtain:

$$
\begin{aligned}
\frac{\partial \widehat{u}}{\partial t}(\xi, t)+\left(\xi^{3}+\xi\right) \widehat{u}(\xi, t)= & \widehat{f}(\xi, t)+\left(\xi^{2}+1\right) g(t) \\
& +\xi u_{x}(0, t)+u_{x x}(0, t),
\end{aligned}
$$

where $\widehat{f}(\xi, t)$ denotes the Laplace transform of $f$ with respect to $x$. Integrating with respect to $t$ yields:

$$
\begin{aligned}
\widehat{u}(\xi, t)= & \mathrm{e}^{-\left(\xi^{3}+\xi\right) t} \widehat{u}_{0}(\xi)+\mathrm{e}^{-\left(\xi^{3}+\xi\right) t} \int_{0}^{t} \mathrm{e}^{\left(\xi^{3}+\xi\right) \tau} \widehat{f}(\xi, \tau) \mathrm{d} \tau \\
& +\mathrm{e}^{-\left(\xi^{3}+\xi\right) t} \int_{0}^{t} \mathrm{e}^{\left(\xi^{3}+\xi\right) \tau}\left(\left(\xi^{2}+1\right) g(\tau)\right. \\
& \left.+\xi u_{x}(0, \tau)+u_{x x}(0, \tau)\right) \mathrm{d} \tau
\end{aligned}
$$

To find $u(x, t)$, we take the inverse Laplace transform of $\widehat{u}(\xi, t)$. If the inverse Laplace transform exists, then the growth rate of $\widehat{u}(\xi, t)$ in terms of $|\xi|$ for $\operatorname{Re}(\xi) \geq 0$ should be at most algebraic, according to Proposition A.3 of Appendix A. To make this requirement precise, we rewrite $\widehat{u}(\xi, t)$ as:

$\widehat{u}(\xi, t)=\mathrm{e}^{-\left(\xi^{3}+\xi\right) t}\left[\widehat{u}_{0}(\xi)+\int_{0}^{\infty} \mathrm{e}^{\left(\xi^{3}+\xi\right) \tau}(\widehat{f}(\xi, \tau)\right.$ 


$$
\begin{aligned}
& \left.\left.+\left(\xi^{2}+1\right) g(\tau)+\xi u_{x}(0, \tau)+u_{x x}(0, \tau)\right) \mathrm{d} \tau\right] \\
& -\int_{t}^{\infty} \mathrm{e}^{-\left(\xi^{3}+\xi\right)(t-\tau)}\left(\widehat{f}(\xi, \tau)+\left(\xi^{2}+1\right) g(\tau)\right. \\
& \left.+\xi u_{x}(0, \tau)+u_{x x}(0, \tau)\right) \mathrm{d} \tau .
\end{aligned}
$$

Therefore, for $\operatorname{Re}(\xi) \geq 0$ and $\operatorname{Re}\left(\xi^{3}+\xi\right)<0$,

$$
\begin{gathered}
\widehat{u}_{0}(\xi)+\int_{0}^{\infty} \mathrm{e}^{\left(\xi^{3}+\xi\right) \tau}\left(\widehat{f}(\xi, \tau)+\left(\xi^{2}+1\right) g(\tau)\right. \\
\left.+\xi u_{x}(0, \tau)+u_{x x}(0, \tau)\right) \mathrm{d} \tau=0 .
\end{gathered}
$$

Setting $\xi^{3}+\xi=-\eta$ implies that:

$$
\begin{gathered}
\widehat{u}_{0}(\xi)+\int_{0}^{\infty} \mathrm{e}^{-\eta \tau}\left(\widehat{f}(\xi, \tau)+\left(\xi^{2}+1\right) g(\tau)\right. \\
\left.+\xi u_{x}(0, \tau)+u_{x x}(0, \tau)\right) \mathrm{d} \tau=0
\end{gathered}
$$

for any $\operatorname{Re}(\eta)>0$ and $\operatorname{Re}(\xi) \geq 0$ satisfying

$\xi^{3}+\xi=-\eta$.

Note that the cubic equation $\xi^{3}+\xi=-\eta$ with $\operatorname{Re}(\eta)>0$ always has two roots $\xi_{1}$ and $\xi_{2}$ with $\operatorname{Re}\left(\xi_{1}\right) \geq 0$ and $\operatorname{Re}\left(\xi_{2}\right) \geq 0$, and a third root $\xi_{3}$ with $\operatorname{Re}\left(\xi_{3}\right)<0$. Therefore, (2.13) is then reduced to the following two equations:

$$
\begin{aligned}
& \widehat{u}_{0}\left(\xi_{j}\right)+\int_{0}^{\infty} \mathrm{e}^{-\eta \tau}\left(\widehat{f}\left(\xi_{j}, \tau\right)+\left(\xi_{j}^{2}+1\right) g(\tau)\right. \\
& \left.\quad+\xi_{j} u_{x}(0, \tau)+u_{x x}(0, \tau)\right) \mathrm{d} \tau=0, \quad j=1,2 .
\end{aligned}
$$

Setting

$\widehat{\widehat{f}}(\xi, \eta)=\int_{0}^{\infty} \mathrm{e}^{-\eta \tau} \widehat{f}(\xi, \tau) \mathrm{d} \tau \quad$ and

$\widehat{g}(\eta)=\int_{0}^{\infty} \mathrm{e}^{-\eta \tau} g(\tau) \mathrm{d} \tau$,

the equations can then be written as:

$\xi_{j} \int_{0}^{\infty} \mathrm{e}^{-\eta \tau} u_{x}(0, \tau) \mathrm{d} \tau+\int_{0}^{\infty} \mathrm{e}^{-\eta \tau} u_{x x}(0, \tau) \mathrm{d} \tau=R\left(\xi_{j}\right)$,

$j=1,2$,

where

$R\left(\xi_{j}\right)=-\widehat{u}_{0}\left(\xi_{j}\right)-\widehat{\widehat{f}}\left(\xi_{j}, \eta\right)-\left(1+\xi_{j}^{2}\right) \widehat{g}(\eta)$.

It then follows from (2.14) that:

$\begin{aligned} \int_{0}^{\infty} \mathrm{e}^{-\eta \tau} u_{x}(0, \tau) \mathrm{d} \tau & =\frac{R\left(\xi_{1}\right)-R\left(\xi_{2}\right)}{\xi_{1}-\xi_{2}}, \\ \int_{0}^{\infty} \mathrm{e}^{-\eta \tau} u_{x x}(0, \tau) \mathrm{d} \tau & =\frac{\xi_{1} R\left(\xi_{2}\right)-\xi_{2} R\left(\xi_{1}\right)}{\xi_{1}-\xi_{2}},\end{aligned}$

which are the Laplace transform of $u_{x}(0, t)$ and $u_{x x}(0, t)$ with respect to $t$. We take the inverse Laplace transforms of these equations to find $u_{x}(0, t)$ and $u_{x x}(0, t)$ and then insert them back in (2.11). Since we need the combination $\xi u_{x}(0, t)+$ $u_{x x}(0, t)$, we consider:

$$
\begin{aligned}
& \int_{0}^{\infty} \mathrm{e}^{-\eta \tau}\left(\xi u_{x}(0, \tau)+u_{x x}(0, \tau)\right) \mathrm{d} \tau \\
& =\xi \frac{R\left(\xi_{1}\right)-R\left(\xi_{2}\right)}{\xi_{1}-\xi_{2}}+\frac{\xi_{1} R\left(\xi_{2}\right)-\xi_{2} R\left(\xi_{1}\right)}{\xi_{1}-\xi_{2}} .
\end{aligned}
$$

Using (2.15), we can sort the terms in (2.16) into three groups:

$$
\int_{0}^{\infty} \mathrm{e}^{-\eta \tau}\left(\xi u_{x}(0, \tau)+u_{x x}(0, \tau)\right) \mathrm{d} \tau=I_{1}+I_{2}+I_{3},
$$

where

$$
\begin{aligned}
I_{1} & =\frac{\left(\xi_{2}-\xi\right) \widehat{u}_{0}\left(\xi_{1}\right)+\left(\xi-\xi_{1}\right) \widehat{u}_{0}\left(\xi_{2}\right)}{\xi_{1}-\xi_{2}}, \\
I_{2} & =\frac{\left(\xi_{2}-\xi\right) \widehat{\widehat{f}}\left(\xi_{1}, \eta\right)+\left(\xi-\xi_{1}\right) \widehat{\widehat{f}}\left(\xi_{2}, \eta\right)}{\xi_{1}-\xi_{2}}, \\
I_{3} & =\left(\xi_{1} \xi_{2}-\xi \xi_{1}-\xi \xi_{2}-1\right) \widehat{g}(\eta) .
\end{aligned}
$$

Taking the inverse Laplace transform of (2.17), we find:

$\xi u_{x}(0, t)+u_{x x}(0, t)=\frac{1}{2 \pi \mathrm{i}} \int_{-\mathrm{i} \infty}^{\mathrm{i} \infty} \mathrm{e}^{\eta t}\left(I_{1}+I_{2}+I_{3}\right) \mathrm{d} \eta$.

Inserting this equation in (2.11) yields:

$\widehat{u}(\xi, t)=J_{1}+J_{2}+J_{3}$,

where

$$
\begin{aligned}
J_{1}= & \mathrm{e}^{-\left(\xi^{3}+\xi\right) t} \widehat{u}_{0}(\xi) \\
& +\int_{0}^{t} \mathrm{e}^{-\left(\xi^{3}+\xi\right)(t-\tau)}\left[\frac{1}{2 \pi \mathrm{i}} \int_{-\mathrm{i} \infty}^{\mathrm{i} \infty} \mathrm{e}^{\eta \tau} I_{1} \mathrm{~d} \eta\right] \mathrm{d} \tau, \\
J_{2}= & \int_{0}^{t} \mathrm{e}^{-\left(\xi^{3}+\xi\right)(t-\tau)}\left[\widehat{f}(\xi, \tau)+\frac{1}{2 \pi \mathrm{i}} \int_{-\mathrm{i} \infty}^{\mathrm{i} \infty} \mathrm{e}^{\eta \tau} I_{2} \mathrm{~d} \eta\right] \mathrm{d} \tau, \\
J_{3}= & \int_{0}^{t} \mathrm{e}^{-\left(\xi^{3}+\xi\right)(t-\tau)}\left[\left(\xi^{2}+1\right) g(\tau)\right. \\
& \left.+\frac{1}{2 \pi \mathrm{i}} \int_{-\mathrm{i} \infty}^{\mathrm{i} \infty} \mathrm{e}^{\eta \tau} I_{3} \mathrm{~d} \eta\right] \mathrm{d} \tau .
\end{aligned}
$$

When $u_{0}, g$ and $f$ satisfy (2.4), the growth rate of $\widehat{u}(\xi, t)$ is at most algebraic and its inverse Laplace transform yields $u$. In order to find a formula for $u$, we simplify the terms above and then take the inverse Laplace transform. For $I_{1}$, we exchange the order of integrals to obtain:

$$
\begin{aligned}
J_{1}= & \mathrm{e}^{-\left(\xi^{3}+\xi\right) t} \widehat{u}_{0}(\xi)+\mathrm{e}^{-\left(\xi^{3}+\xi\right) t} \frac{1}{2 \pi \mathrm{i}} \\
& \times \int_{-\mathrm{i} \infty}^{\mathrm{i} \infty} \frac{\mathrm{e}^{\left(\xi^{3}+\xi\right) t+\eta t}-1}{\xi^{3}+\xi+\eta} I_{1} \mathrm{~d} \eta .
\end{aligned}
$$

We now apply Cauchy's theorem to show that

$\int_{-\mathrm{i} \infty}^{\mathrm{i} \infty} \frac{I_{1}}{\xi^{3}+\xi+\eta} \mathrm{d} \eta=0$.

The idea is to approximate the integral by

$\int_{-\mathrm{i} R}^{\mathrm{i} R} \frac{I_{1}}{\xi^{3}+\xi+\eta} \mathrm{d} \eta$

and then close the contour with a semicircle in the right halfplane. Since there are no poles of the integrand inside this contour that the integral vanishes due to Cauchy's theorem. It then remains to show that the integrand decays sufficiently fast in $\eta$ as $|\eta| \rightarrow \infty$. For this purpose, we insert:

$\widehat{u}_{0}\left(\xi_{i}\right)=\int \mathrm{e}^{-\xi_{i} x} u_{0}(x) \mathrm{d} x, \quad i=1,2$ 
in (2.18) to obtain

$I_{1}=\int Q(\xi, \eta, x) u_{0}(x) \mathrm{d} x$

where

$Q(\xi, \eta, x)=\frac{\left(\xi_{2}(\eta)-\xi\right) \mathrm{e}^{-\xi_{1}(\eta) x}+\left(\xi-\xi_{1}(\eta)\right) \mathrm{e}^{-\xi_{2}(\eta) x}}{\xi_{1}(\eta)-\xi_{2}(\eta)}$.

For $|\eta|$ sufficiently large, $\xi_{1}(\eta)$ and $\xi_{2}(\eta)$ obeys the asymptotics:

$\xi_{1}(\eta)=|\eta|^{\frac{1}{3}} \mathrm{e}^{\mathrm{i} \frac{\theta+\pi}{3}}+o\left(|\eta|^{\frac{1}{3}}\right)$,

$\xi_{2}(\eta)=|\eta|^{\frac{1}{3}} \mathrm{e}^{\mathrm{i} \frac{\theta+5 \pi}{3}}+o\left(|\eta|^{\frac{1}{3}}\right)$

in the case when $\eta=|\eta| \mathrm{e}^{\mathrm{i} \theta}$ with $\theta \in[0, \pi / 2)$, and

$\xi_{1}(\eta)=|\eta|^{\frac{1}{3}} \mathrm{e}^{\mathrm{i} \frac{\theta+5 \pi}{3}}+o\left(|\eta|^{\frac{1}{3}}\right)$,

$\xi_{2}(\eta)=|\eta|^{\frac{1}{3}} \mathrm{e}^{\mathrm{i} \frac{\theta+3 \pi}{3}}+o\left(|\eta|^{\frac{1}{3}}\right)$

in the case when $\eta=|\eta| \mathrm{e}^{\mathrm{i} \theta}$ with $\theta \in(3 \pi / 2,2 \pi]$. In both cases,

$|Q(\xi, \eta, x)| \leq C(\xi) \mathrm{e}^{-C|\eta|^{\frac{1}{3}} x}$

holds for any $\operatorname{Re}(\xi) \geq 0, \operatorname{Re}(\eta)>0$ and $x>0$. When $u_{0} \in$ $L^{1}(0, \infty)$, we conclude that the integral in (2.20) vanishes.

$J_{1}$ is then reduced to

$J_{1}=\mathrm{e}^{-\left(\xi^{3}+\xi\right) t} \widehat{u}_{0}(\xi)+\frac{1}{2 \pi \mathrm{i}} \int_{-\mathrm{i} \infty}^{\mathrm{i} \infty} \frac{\mathrm{e}^{\eta t} I_{1}}{\xi^{3}+\xi+\eta} \mathrm{d} \eta$.

Similarly, $J_{2}$ and $J_{3}$ can be simplified to

$$
\begin{aligned}
J_{2}= & \int_{0}^{t} \mathrm{e}^{-\left(\xi^{3}+\xi\right)(t-\tau)} \widehat{f}(\xi, \tau) \mathrm{d} \tau \\
& +\frac{1}{2 \pi \mathrm{i}} \int_{-\mathrm{i} \infty}^{\mathrm{i} \infty} \frac{\mathrm{e}^{\eta t} I_{2}}{\xi^{3}+\xi+\eta} \mathrm{d} \eta, \\
J_{3}= & \int_{0}^{t} \mathrm{e}^{-\left(\xi^{3}+\xi\right)(t-\tau)}\left(\xi^{2}+1\right) g(\tau) \mathrm{d} \tau \\
& +\frac{1}{2 \pi \mathrm{i}} \int_{-\mathrm{i} \infty}^{\mathrm{i} \infty} \frac{\mathrm{e}^{\eta t} I_{3}}{\xi^{3}+\xi+\eta} \mathrm{d} \eta .
\end{aligned}
$$

Inserting (2.21)-(2.23) in (2.19) and then taking the inverse Laplace transform of (2.19), we obtain

$u(x, t)=K_{1}+K_{2}+K_{3}$,

where $K_{m}$ denotes the inverse Laplace transform of $J_{m}$, namely:

$K_{m}=\frac{1}{2 \pi \mathrm{i}} \int_{-\mathrm{i} \infty}^{\mathrm{i} \infty} \mathrm{e}^{\xi x} J_{m} \mathrm{~d} \xi, \quad m=1,2,3$.

Since $\widehat{u}_{0}$ is the Laplace transform of $u_{0}$, namely:

$\widehat{u}_{0}(\xi)=\int_{0}^{\infty} \mathrm{e}^{-\xi y} u_{0}(y) \mathrm{d} y$

we obtain after exchanging the order of integrals

$K_{1}=\int_{0}^{\infty} \Gamma(x, y, t) u_{0}(y) \mathrm{d} y$, where the kernel function $\Gamma$ is given by

$$
\begin{aligned}
& \Gamma(x, y, t)=\frac{1}{2 \pi \mathrm{i}} \int_{-\mathrm{i} \infty}^{\mathrm{i} \infty} \mathrm{e}^{\xi(x-y)} \mathrm{e}^{-\left(\xi^{3}+\xi\right) t} \mathrm{~d} \xi \\
& +\frac{1}{2 \pi \mathrm{i}} \int_{-\mathrm{i} \infty}^{\mathrm{i} \infty} \mathrm{e}^{\xi x}\left[\frac{1}{2 \pi \mathrm{i}} \int_{-\mathrm{i} \infty}^{\mathrm{i} \infty} \frac{\mathrm{e}^{\eta t}}{\xi^{3}+\xi+\eta}\right. \\
& \left.\quad \times\left(\frac{\left(\xi_{2}(\eta)-\xi\right) \mathrm{e}^{-\xi_{1}(\eta) y}+\left(\xi-\xi_{1}(\eta)\right) \mathrm{e}^{-\xi_{2}(\eta) y}}{\xi_{1}(\eta)-\xi_{2}(\eta)}\right) \mathrm{d} \eta\right] \mathrm{d} \xi
\end{aligned}
$$

Similarly, $K_{2}$ can be represented as follows:

$K_{2}=\int_{0}^{t} \int_{0}^{\infty} \Gamma(x, y, t-\tau) f(y, \tau) \mathrm{d} y \mathrm{~d} \tau$.

We now find a compact expression for $K_{3}$. Inserting $I_{3}$ in $J_{3}$ yields

$$
\begin{aligned}
J_{3}= & \int_{0}^{t} \mathrm{e}^{-\left(\xi^{3}+\xi\right)(t-\tau)}\left(\xi^{2}+1\right) g(\tau) \mathrm{d} \tau \\
& +\frac{1}{2 \pi \mathrm{i}} \int_{-\mathrm{i} \infty}^{\mathrm{i} \infty} \frac{\mathrm{e}^{\eta t}}{\xi^{3}+\xi+\eta}\left(\xi_{1} \xi_{2}-\xi\left(\xi_{1}+\xi_{2}\right)-1\right) \widehat{g}(\eta) \mathrm{d} \eta .
\end{aligned}
$$

Noting that

$\frac{1}{2 \pi \mathrm{i}} \int_{-\mathrm{i} \infty}^{\mathrm{i} \infty} \mathrm{e}^{\eta \tau} \widehat{g}(\eta) \mathrm{d} \eta=g(\tau)$,

we use the basic property of the Laplace transform $\mathcal{L}^{-1}(\widehat{F} \widehat{G})=$ $F * G$ to find

$$
\begin{aligned}
J_{3} & =\int_{0}^{t} \mathrm{e}^{-\left(\xi^{3}+\xi\right)(t-\tau)}\left(\xi^{2}+1\right) g(\tau) \mathrm{d} \tau \\
& +\int_{0}^{t}\left[\frac{1}{2 \pi \mathrm{i}} \int_{-\mathrm{i} \infty}^{\mathrm{i} \infty} \frac{\mathrm{e}^{\eta(t-\tau)}}{\xi^{3}+\xi+\eta}\left(\xi_{1} \xi_{2}-\xi\left(\xi_{1}+\xi_{2}\right)-1\right) \mathrm{d} \eta\right] \\
& \times g(\tau) \mathrm{d} \tau .
\end{aligned}
$$

Therefore, $K_{3}$, the inverse Laplace transform of $J_{3}$, can be represented as

$K_{3}=\int_{0}^{t} \Phi(x, t-\tau) g(\tau) \mathrm{d} \tau$,

where

$$
\begin{aligned}
\Phi(x, t)= & \frac{1}{2 \pi \mathrm{i}} \int_{-\mathrm{i} \infty}^{\mathrm{i} \infty} \mathrm{e}^{\xi x} \mathrm{e}^{-\left(\xi^{3}+\xi\right) t}\left(\xi^{2}+1\right) \mathrm{d} \xi \\
& +\frac{1}{2 \pi \mathrm{i}} \int_{-\mathrm{i} \infty}^{\mathrm{i} \infty} \mathrm{e}^{\xi x}\left[\frac{1}{2 \pi \mathrm{i}} \int_{-\mathrm{i} \infty}^{\mathrm{i} \infty} \frac{\mathrm{e}^{\eta t}}{\xi^{3}+\xi+\eta}\right. \\
& \left.\times\left(\xi_{1} \xi_{2}-\xi\left(\xi_{1}+\xi_{2}\right)-1\right) \mathrm{d} \eta\right] \mathrm{d} \xi
\end{aligned}
$$

We further simplify $\Gamma$ and $\Phi$. We need to compute the integrals

$\frac{1}{2 \pi \mathrm{i}} \int_{-\mathrm{i} \infty}^{\mathrm{i} \infty} \frac{\mathrm{e}^{\xi x}}{\xi^{3}+\xi+\eta} \mathrm{d} \xi$ and $\frac{1}{2 \pi \mathrm{i}} \int_{-\mathrm{i} \infty}^{\mathrm{i} \infty} \frac{\mathrm{e}^{\xi x} \xi}{\xi^{3}+\xi+\eta} \mathrm{d} \xi$.

Fix $\eta$ with $\operatorname{Re}(\eta)>0$ and let $R>0$ be large. Consider the contour $\Omega$ consisting of the Bromwich path from $-\mathrm{i} R$ to $\mathrm{i} R$ 
and the connecting left half circle. According to the Residue theorem, we have:

$$
\begin{aligned}
\frac{1}{2 \pi \mathrm{i}} \int_{-\mathrm{i} \infty}^{\mathrm{i} \infty} \frac{\mathrm{e}^{\xi x}}{\xi^{3}+\xi+\eta} \mathrm{d} \xi & =\lim _{R \rightarrow \infty} \frac{1}{2 \pi \mathrm{i}} \int_{-\mathrm{i} R}^{\mathrm{i} R} \frac{\mathrm{e}^{\xi x}}{\xi^{3}+\xi+\eta} \mathrm{d} \xi \\
& =\lim _{R \rightarrow \infty} \frac{1}{2 \pi \mathrm{i}} \int_{\Omega} \frac{\mathrm{e}^{\xi x}}{\xi^{3}+\xi+\eta} \mathrm{d} \xi \\
& =\frac{\mathrm{e}^{\xi_{3}(\eta) x}}{3 \xi_{3}^{2}(\eta)+1} .
\end{aligned}
$$

Using the relation $\left(3 \xi_{3}^{2}(\eta)+1\right) \xi_{3}^{\prime}(\eta)=-1$, we have

$\frac{1}{2 \pi \mathrm{i}} \int_{-\mathrm{i} \infty}^{\mathrm{i} \infty} \frac{\mathrm{e}^{\xi x}}{\xi^{3}+\xi+\eta} \mathrm{d} \xi=-\mathrm{e}^{\xi_{3}(\eta) x} \xi_{3}^{\prime}(\eta)$.

Similarly,

$\frac{1}{2 \pi \mathrm{i}} \int_{-\mathrm{i} \infty}^{\mathrm{i} \infty} \frac{\mathrm{e}^{\xi x} \xi}{\xi^{3}+\xi+\eta} \mathrm{d} \xi=-\mathrm{e}^{\xi_{3}(\eta) x} \xi_{3}^{\prime}(\eta) \xi_{3}(\eta)$.

Inserting these relations in the second integral of (2.24), we obtain

$$
\begin{aligned}
& \Gamma(x, y, t)=\frac{1}{2 \pi \mathrm{i}} \int_{-\mathrm{i} \infty}^{\mathrm{i} \infty} \mathrm{e}^{\xi(x-y)} \mathrm{e}^{-\left(\xi^{3}+\xi\right) t} \mathrm{~d} \xi \\
& +\frac{1}{2 \pi \mathrm{i}} \int_{-\mathrm{i} \infty}^{\mathrm{i} \infty} \mathrm{e}^{\eta t} \mathrm{e}^{\xi_{3} x} \xi_{3}^{\prime} \frac{\left(\xi_{3}-\xi_{2}\right) \mathrm{e}^{-\xi_{1} y}+\left(\xi_{1}-\xi_{3}\right) \mathrm{e}^{-\xi_{2} y}}{\xi_{1}-\xi_{2}} \mathrm{~d} \eta .
\end{aligned}
$$

The kernel function $\Phi$ can be similarly simplified. In fact,

$$
\begin{aligned}
\Phi(x, t)= & \frac{1}{2 \pi \mathrm{i}} \int_{-\mathrm{i} \infty}^{\mathrm{i} \infty} \mathrm{e}^{\xi x} \mathrm{e}^{-\left(\xi^{3}+\xi\right) t}\left(\xi^{2}+1\right) \mathrm{d} \xi \\
& +\frac{1}{2 \pi \mathrm{i}} \int_{-\mathrm{i} \infty}^{\mathrm{i} \infty} \mathrm{e}^{\eta t} \mathrm{e}^{\xi_{3} x} \xi_{3}^{\prime}\left(\xi_{1} \xi_{3}+\xi_{2} \xi_{3}-\xi_{1} \xi_{2}+1\right) \mathrm{d} \eta .
\end{aligned}
$$

The roots $\xi_{1}, \xi_{2}$ and $\xi_{3}$ of $\xi^{3}+\xi+\eta=0$ satisfy

$$
\begin{aligned}
& \xi_{1}+\xi_{2}+\xi_{3}=0, \quad \xi_{1} \xi_{2}+\xi_{1} \xi_{3}+\xi_{2} \xi_{3}=1, \\
& \xi_{1} \xi_{2} \xi_{3}=-\eta, \quad \xi_{1}^{\prime}\left(\xi_{1}-\xi_{2}\right)\left(\xi_{1}-\xi_{3}\right)=-1, \\
& \xi_{2}^{\prime}\left(\xi_{2}-\xi_{1}\right)\left(\xi_{2}-\xi_{3}\right)=-1, \\
& \xi_{3}^{\prime}\left(\xi_{3}-\xi_{1}\right)\left(\xi_{3}-\xi_{2}\right)=-1 .
\end{aligned}
$$

The first three equations reflect the relationship between the roots and the coefficients and the second three are easy consequences of the first three. Differentiating the equation $\xi^{3}+\xi+\eta=0$ with respect to $\eta$ and setting $\xi=\xi_{1}$, we get

$\xi_{1}^{\prime}\left(3 \xi_{1}^{2}+1\right)=-1$.

Combining the first two equations yields $3 \xi_{1}^{2}+1=\left(\xi_{1}-\right.$ $\left.\xi_{2}\right)\left(\xi_{1}-\xi_{3}\right)$ and thus

$\xi_{1}^{\prime}\left(\xi_{1}-\xi_{2}\right)\left(\xi_{1}-\xi_{3}\right)=-1$.

Using these equations, we find

$$
\begin{aligned}
\Gamma(x, y, t)= & \frac{1}{2 \pi \mathrm{i}} \int_{-\mathrm{i} \infty}^{\mathrm{i} \infty} \mathrm{e}^{\xi(x-y)} \mathrm{e}^{-\left(\xi^{3}+\xi\right) t} \mathrm{~d} \xi \\
& -\frac{1}{2 \pi \mathrm{i}} \int_{-\mathrm{i} \infty}^{\mathrm{i} \infty} \mathrm{e}^{\eta t} \mathrm{e}^{\xi_{3} x}\left(\mathrm{e}^{-\xi_{1} y} \xi_{1}^{\prime}+\mathrm{e}^{-\xi_{2} y} \xi_{2}^{\prime}\right) \mathrm{d} \eta,
\end{aligned}
$$

$$
\begin{aligned}
\Phi(x, t)= & \frac{1}{2 \pi \mathrm{i}} \int_{-\mathrm{i} \infty}^{\mathrm{i} \infty} \mathrm{e}^{\xi x} \mathrm{e}^{-\left(\xi^{3}+\xi\right) t}\left(\xi^{2}+1\right) \mathrm{d} \xi \\
& -\frac{1}{\pi \mathrm{i}} \int_{-\mathrm{i} \infty}^{\mathrm{i} \infty} \mathrm{e}^{\eta t} \mathrm{e}^{\xi_{3} x} \xi_{3}^{\prime} \xi_{3}^{2} \mathrm{~d} \eta .
\end{aligned}
$$

This completes the proof of Theorem 2.1.

Finally we derive the compact form of $\Gamma$ and $\Phi$ given in Corollary 2.2. By Cauchy's formula,

$\frac{1}{2 \pi \mathrm{i}} \int_{-\mathrm{i} \infty}^{\mathrm{i} \infty} \frac{\mathrm{e}^{\eta t}}{\xi^{3}+\xi+\eta} \mathrm{d} \eta=\mathrm{e}^{-\left(\xi^{3}+\xi\right) t}$.

Therefore,

$$
\begin{aligned}
& \frac{1}{2 \pi \mathrm{i}} \int_{-\mathrm{i} \infty}^{\mathrm{i} \infty} \mathrm{e}^{\xi(x-y)} \mathrm{e}^{-\left(\xi^{3}+\xi\right) t} \mathrm{~d} \xi \\
& \quad=\frac{1}{2 \pi \mathrm{i}} \int_{-\mathrm{i} \infty}^{\mathrm{i} \infty} \mathrm{e}^{\xi(x-y)}\left[\frac{1}{2 \pi \mathrm{i}} \int_{-\mathrm{i} \infty}^{\mathrm{i} \infty} \frac{\mathrm{e}^{\eta t}}{\xi^{3}+\xi+\eta} \mathrm{d} \eta\right] \mathrm{d} \xi
\end{aligned}
$$

Changing the order of the integrals and applying the Residue Theorem, we have

$\frac{1}{2 \pi \mathrm{i}} \int_{-\mathrm{i} \infty}^{\mathrm{i} \infty} \mathrm{e}^{\xi(x-y)} \mathrm{e}^{-\left(\xi^{3}+\xi\right) t} \mathrm{~d} \xi=-\frac{1}{2 \pi \mathrm{i}} \int_{-\mathrm{i} \infty}^{\mathrm{i} \infty} \mathrm{e}^{\eta t} \mathrm{e}^{\xi_{3}(x-y)} \xi_{3}^{\prime} \mathrm{d} \eta$.

Inserting this equation in (2.5) then yields (2.7) of Corollary 2.2. Similarly, we have

$$
\begin{aligned}
& \frac{1}{2 \pi \mathrm{i}} \int_{-\mathrm{i} \infty}^{\mathrm{i} \infty} \mathrm{e}^{\xi x} \mathrm{e}^{-\left(\xi^{3}+\xi\right) t}\left(\xi^{2}+1\right) \mathrm{d} \xi \\
& \quad=-\frac{1}{2 \pi \mathrm{i}} \int_{-\mathrm{i} \infty}^{\mathrm{i} \infty} \mathrm{e}^{\eta t} \mathrm{e}^{\xi_{3} x}\left(\xi_{3}^{2}+1\right) \xi_{3}^{\prime} \mathrm{d} \eta
\end{aligned}
$$

Combining this equation with (2.6) then leads to (2.8).

\subsection{Time-decay estimates for the kernel functions}

We prove the large-time asymptotics of $\Gamma$ and $\Phi$ stated in Theorem 2.3 by the method of stationary phase.

Proof of Theorem 2.3. We start with the first integral in $\Gamma$. For notational convenience, we label this integral as $\Gamma_{1}$. Letting $\xi=\mathrm{i} \sigma$, we have

$\Gamma_{1}=\frac{1}{2 \pi} \int_{-\infty}^{\infty} \mathrm{e}^{\mathrm{i} \sigma(x-y)} \mathrm{e}^{\mathrm{i}\left(\sigma^{3}-\sigma\right) t} \mathrm{~d} \sigma=\Gamma_{11}+\Gamma_{12}$

where

$\Gamma_{11}=\int_{0}^{\infty} \mathrm{e}^{\mathrm{i} \sigma(x-y)} \mathrm{e}^{\mathrm{i}\left(\sigma^{3}-\sigma\right) t} \mathrm{~d} \sigma \quad$ and

$\Gamma_{12}=\int_{-\infty}^{0} \mathrm{e}^{\mathrm{i} \sigma(x-y)} \mathrm{e}^{\mathrm{i}\left(\sigma^{3}-\sigma\right) t} \mathrm{~d} \sigma$.

Although the integrands in these integrals do not die away as $\sigma \rightarrow \pm \infty$, the integrals converge because the increasingly rapid oscillations. Since $\Gamma_{12}$ is the complex conjugate of $\Gamma_{11}$, it then suffices to consider $\Gamma_{11}$. We further split $\Gamma_{11}$ into two integrals:

$$
\Gamma_{11}=\int_{0}^{\frac{1}{\sqrt{3}}} \mathrm{e}^{\mathrm{i} \sigma(x-y)} \mathrm{e}^{\mathrm{i}\left(\sigma^{3}-\sigma\right) t} \mathrm{~d} \sigma+\int_{\frac{1}{\sqrt{3}}}^{\infty} \mathrm{e}^{\mathrm{i} \sigma(x-y)} \mathrm{e}^{\mathrm{i}\left(\sigma^{3}-\sigma\right) t} \mathrm{~d} \sigma .
$$


To apply the method of stationary phase, we further change the variable $\sigma$ to $-\sigma$ in the first integral:

$\int_{0}^{\frac{1}{\sqrt{3}}} \mathrm{e}^{\mathrm{i}\left(\sigma^{3}-\sigma\right) t} \mathrm{e}^{\mathrm{i} \sigma x} \mathrm{~d} \sigma=\int_{-\frac{1}{\sqrt{3}}}^{0} \mathrm{e}^{\mathrm{i} t\left(\sigma-\sigma^{3}\right)} \mathrm{e}^{-\mathrm{i} \sigma x} \mathrm{~d} \sigma$.

Direct applications of the method of stationary phase allows the inferences:

$$
\begin{aligned}
& \int_{-\frac{1}{\sqrt{3}}}^{0} \mathrm{e}^{\mathrm{i}\left(\sigma-\sigma^{3}\right) t} \mathrm{e}^{-\mathrm{i} \sigma(x-y)} \mathrm{d} \sigma \sim \frac{\sqrt{\pi} \mathrm{e}^{\frac{\pi \mathrm{i}}{4}} \mathrm{e}^{\frac{\mathrm{i}}{\sqrt{3}}\left(x-y-\frac{2}{3} t\right)}}{2 \sqrt[4]{3} \sqrt{t}}, \\
& \int_{\frac{1}{\sqrt{3}}}^{\infty} \mathrm{e}^{\mathrm{i}\left(\sigma^{3}-\sigma\right) t} \mathrm{e}^{\mathrm{i} \sigma(x-y)} \mathrm{d} \sigma \sim \frac{\sqrt{\pi} \mathrm{e}^{\frac{\pi \mathrm{i}}{4}} \mathrm{e}^{\frac{\mathrm{i}}{\sqrt{3}}\left(x-y-\frac{2}{3} t\right)}}{2 \sqrt[4]{3} \sqrt{t}}
\end{aligned}
$$

as $t \rightarrow \infty$. For readers' convenience, the method of stationary phase are recalled in Appendix B and the details leading to these asymptotic estimates are also provided in Appendix B.

We now estimate the second part in $\Gamma$ :

$\Gamma_{2} \equiv \frac{1}{2 \pi \mathrm{i}} \int_{-\mathrm{i} \infty}^{\mathrm{i} \infty} \mathrm{e}^{\eta t} \mathrm{e}^{\xi_{3} x}\left(\mathrm{e}^{-\xi_{1} y} \xi_{1}^{\prime}+\mathrm{e}^{-\xi_{2} y} \xi_{2}^{\prime}\right) \mathrm{d} \eta$.

We first represent $\xi_{1}$ and $\xi_{2}$ in terms of $\xi_{3}$. It follows from (2.26) that:

$\xi_{1}=\frac{-\xi_{3}-\sqrt{-4-3 \xi_{3}^{2}}}{2}, \quad \xi_{2}=\frac{-\xi_{3}+\sqrt{-4-3 \xi_{3}^{2}}}{2}$.

Making the substitution $\eta=\mathrm{i} \sigma$, we obtain:

$$
\begin{aligned}
\Gamma_{2}= & \frac{1}{2 \pi} \int_{-\infty}^{\infty} \mathrm{e}^{\mathrm{i} \sigma t} \mathrm{e}^{\xi_{3} x} \\
& \times\left[\mathrm{e}^{\frac{1}{2}\left(\xi_{3}+\sqrt{-4-3 \xi_{3}^{2}}\right) y}\left(-\frac{1}{2}+\frac{3 \xi_{3}}{2 \sqrt{-4-3 \xi_{3}^{2}}}\right)\right. \\
& \left.+\mathrm{e}^{\frac{1}{2}\left(\xi_{3}-\sqrt{-4-3 \xi_{3}^{2}}\right) y}\left(-\frac{1}{2}-\frac{3 \xi_{3}}{2 \sqrt{-4-3 \xi_{3}^{2}}}\right)\right] \mathrm{d} \sigma .
\end{aligned}
$$

To analyse this integral, we break it into four parts $\Gamma_{21}, \Gamma_{22}, \Gamma_{23}$ and $\Gamma_{24}$ representing the integrals over the intervals:

$$
\begin{aligned}
& \left(-\infty,-\frac{2}{3 \sqrt{3}}\right], \quad\left(-\frac{2}{3 \sqrt{3}}, 0\right], \\
& \left(0, \frac{2}{3 \sqrt{3}}\right], \quad\left(\frac{2}{3 \sqrt{3}}, \infty\right),
\end{aligned}
$$

respectively. We will consider $\Gamma_{22}$ and $\Gamma_{24}$ since $\Gamma_{21}$ and $\Gamma_{23}$ can be similarly treated. Since $\xi_{3}(\mathrm{i} \sigma)$ is the root of $\xi^{3}+\xi+\mathrm{i} \sigma=$ 0 with a negative or zero real part, its real part $s$ and imaginary part $\rho$ satisfies:

$s=-\sqrt{3 \rho^{2}-1}, \quad 8 \rho^{3}-2 \rho+\sigma=0$

for $\sigma \in\left(-\infty,-\frac{2}{3 \sqrt{3}}\right], \rho \geq \frac{1}{\sqrt{3}}$;

$s=0, \quad \rho^{3}-\rho-\sigma=0$

for $\sigma \in\left[-\frac{2}{3 \sqrt{3}}, \frac{2}{3 \sqrt{3}}\right], \rho \in\left[-\frac{1}{\sqrt{3}}, \frac{1}{\sqrt{3}}\right]$; $s=-\sqrt{3 \rho^{2}-1}, \quad 8 \rho^{3}-2 \rho+\sigma=0$

for $\sigma \in\left[\frac{2}{3 \sqrt{3}}, \infty\right), \rho \leq-\frac{1}{\sqrt{3}}$.

To consider $\Gamma_{22}$, we make the change of variables

$\sigma=r-r^{3}, \quad r \in\left[-\frac{1}{\sqrt{3}}, 0\right]$.

Noticing that $\sigma$ is a monotonic function of $r$, we have:

$\rho(r)=-r, \quad \rho^{\prime}(\sigma)=\frac{1}{3 \rho^{2}-1}=\frac{1}{3 r^{2}-1}$,

$\mathrm{d} \sigma=\left(1-3 r^{2}\right) \mathrm{d} r$

and $\Gamma_{22}$ becomes

$$
\begin{aligned}
\Gamma_{22}= & -\int_{-\frac{1}{\sqrt{3}}}^{0} \mathrm{e}^{\mathrm{i}\left(r-r^{3}\right) t} \mathrm{e}^{-\mathrm{i} r x} \\
& \times\left[\mathrm{e}^{\frac{\mathrm{i}}{2}\left(-r+\sqrt{4-3 r^{2}}\right) y}\left(-\frac{1}{2}-\frac{3 r}{2 \sqrt{4-3 r^{2}}}\right)\right. \\
& \left.+\mathrm{e}^{\frac{\mathrm{i}}{2}\left(-r-\sqrt{4-3 r^{2}}\right) y}\left(-\frac{1}{2}+\frac{3 r}{2 \sqrt{4-3 r^{2}}}\right)\right] \mathrm{d} r .
\end{aligned}
$$

It then follows from the theory of stationary phase that

$\Gamma_{22} \sim \frac{\sqrt{\pi} \mathrm{e}^{\mathrm{i}\left(\frac{\pi}{4}+\frac{x}{\sqrt{3}}-\frac{y}{\sqrt{3}}-\frac{2}{3 \sqrt{3}} t\right)}}{2 \sqrt[4]{3} \sqrt{t}}$

as $t \rightarrow \infty$.

We now consider $\Gamma_{24}$ :

$\Gamma_{24}=\int_{\frac{2}{3 \sqrt{3}}}^{\infty} \mathrm{e}^{\mathrm{i} \sigma t} \mathrm{e}^{\xi_{3} x}\left(\mathrm{e}^{-\xi_{1} y} \xi_{1}^{\prime}+\mathrm{e}^{-\xi_{2} y} \xi_{2}^{\prime}\right) \mathrm{d} \sigma$.

Recall that for $\sigma \in\left[\frac{2}{3 \sqrt{3}}, \infty\right)$,

$\xi_{3}=s+\mathrm{i} \rho \quad$ with $s=-\sqrt{3 \rho^{2}-1}$ and

$8 \rho^{3}-2 \rho+\sigma=0$

and

$\xi_{1}=-s+\mathrm{i} \rho \quad$ and $\quad \xi_{2}=-2 \mathrm{i} \rho$.

We make the change of variables in (2.29),

$\sigma=8 z^{3}-2 z, \quad z \in\left[\frac{1}{\sqrt{3}}, \infty\right)$.

Since $\sigma$ is a monotonic function of $z$, we have

$\rho(z)=-z, \quad \xi_{3}=-\sqrt{3 z^{2}-1}-\mathrm{i} z$,

$\xi_{1}=\sqrt{3 z^{2}-1}-\mathrm{i} z, \quad \xi_{2}=2 \mathrm{i} z$.

Thus,

$$
\begin{aligned}
\Gamma_{24}= & \int_{\frac{1}{\sqrt{3}}}^{\infty} \mathrm{e}^{\mathrm{i}\left(8 z^{3}-2 z\right) t} \mathrm{e}^{\left(-\sqrt{3 z^{2}-1}-\mathrm{i} z\right) x} \mathrm{e}^{\left(-\sqrt{3 z^{2}-1}+\mathrm{i} z\right) y} \\
& \times\left(\frac{3 z}{\sqrt{3 z^{2}-1}}-\mathrm{i}\right) \mathrm{d} z \\
& +\int_{\frac{1}{\sqrt{3}}}^{\infty} \mathrm{e}^{\mathrm{i}\left(8 z^{3}-2 z\right) t} \mathrm{e}^{\left(-\sqrt{3 z^{2}-1}-\mathrm{i} z\right) x} \mathrm{e}^{-2 \mathrm{i} z y}(-2 \mathrm{i}) \mathrm{d} z
\end{aligned}
$$


As in Appendix B, the theory of stationary phase yields:

$\Gamma_{24} \sim \frac{\sqrt{\pi} \mathrm{e}^{\mathrm{i}\left(\frac{\pi}{4}-\frac{x}{\sqrt{3}}+\frac{y}{\sqrt{3}}+\frac{2}{3 \sqrt{3}} t\right)}}{2 \sqrt[4]{3} \sqrt{t}}+\frac{\sqrt{\pi} \mathrm{e}^{\mathrm{i}\left(\frac{\pi}{4}-\frac{x}{\sqrt{3}}-\frac{2 y}{\sqrt{3}}+\frac{2}{3 \sqrt{3}} t\right)}}{3 t}$

as $t \rightarrow \infty$. Putting together all these estimates yields (2.9).

We now derive (2.10). Making the substitution $\xi=\mathrm{i} \sigma$ in $\Phi$ (defined in (2.8)), we obtain

$$
\begin{aligned}
\Phi(x, t) & =\frac{1}{2 \pi} \int_{-\infty}^{\infty} \mathrm{e}^{\mathrm{i} \sigma x} \mathrm{e}^{\mathrm{i}\left(\sigma^{3}-\sigma\right) t}\left(1-3 \sigma^{2}\right) \mathrm{d} \sigma \\
& =\frac{\mathrm{i}}{2 \pi t} \int_{-\infty}^{\infty} \mathrm{e}^{\mathrm{i} \sigma x} \frac{\mathrm{d}}{\mathrm{d} \sigma} \mathrm{e}^{\mathrm{i}\left(\sigma^{3}-\sigma\right) t} \mathrm{~d} \sigma .
\end{aligned}
$$

The last expression is interpreted in the sense of distributions. According to a property of the Fourier transform on the derivatives of a function,

$\Phi(x, t)=\frac{x}{2 \pi t} \int_{-\infty}^{\infty} \mathrm{e}^{\mathrm{i} \sigma x} \mathrm{e}^{\mathrm{i}\left(\sigma^{3}-\sigma\right) t} \mathrm{~d} \sigma$.

To further analyse the large-time behaviour of $\Phi$, we decompose the integral above into three parts:

$$
\begin{aligned}
& \int_{-\infty}^{\infty} \mathrm{e}^{\mathrm{i} \sigma x} \mathrm{e}^{\mathrm{i}\left(\sigma^{3}-\sigma\right) t} \mathrm{~d} \sigma \\
& =\left(\int_{-\infty}^{-\frac{1}{\sqrt{3}}}+\int_{-\frac{1}{\sqrt{3}}}^{\frac{1}{\sqrt{3}}}+\int_{\frac{1}{\sqrt{3}}}^{\infty}\right) \mathrm{e}^{\mathrm{i} p(\sigma) t} q(\sigma) \mathrm{d} \sigma,
\end{aligned}
$$

where $p(\sigma)=\sigma^{3}-\sigma$ and $q(\sigma)=\mathrm{e}^{\mathrm{i} \sigma x}$. We then apply the theory of stationary phase to each part. For example:

$\int_{\frac{1}{\sqrt{3}}}^{\infty} \mathrm{e}^{\mathrm{i} p(\sigma) t} q(\sigma) \mathrm{d} \sigma \sim \frac{\sqrt{\pi} \mathrm{e}^{\mathrm{i}\left(\frac{\pi}{4}+\frac{x}{\sqrt{3}}-\frac{2}{3 \sqrt{3}} t\right)}}{2 \sqrt[4]{3} \sqrt{t}}$

as $t \rightarrow \infty$. Therefore, as $t \rightarrow \infty$,

$\Phi(x, t) \sim \frac{x}{2 \sqrt{\pi} \sqrt[4]{3} t^{3 / 2}}\left(\mathrm{e}^{\mathrm{i}\left(\frac{\pi}{4}+\frac{x}{\sqrt{3}}-\frac{2}{3 \sqrt{3}} t\right)}+\mathrm{e}^{\mathrm{i}\left(\frac{\pi}{4}-\frac{x}{\sqrt{3}}+\frac{2}{3 \sqrt{3}} t\right)}\right)$.

This completes the derivation of (2.10).

\subsection{Eventual periodicity}

Let $f \equiv 0$ and $u_{0} \in L^{1}(0, \infty)$. Let $g \in C^{1}(0, \infty)$ be a periodic function of period $T$. Let $u$ the corresponding solution of (2.1). Consider the sequence $\{u(x, n T+t)\}$ and we show that

$\lim _{n \rightarrow \infty} u(x, n T+t)=u_{\infty}(x, t)$

for some function $u_{\infty}$ that is periodic of period $T$ and satisfies $u_{\infty}(0, t)=g(t)$. By Theorem 2.1, $u$ can be represented as

$$
\begin{aligned}
u(x, n T+t)= & \int_{0}^{\infty} \Gamma(x, y, n T+t) u_{0}(y) \mathrm{d} y \\
& +\int_{0}^{n T+t} \Phi(x, n T+t-\tau) g(\tau) \mathrm{d} \tau .
\end{aligned}
$$

Using the estimates in Theorem 2.3, we have

$\left|\int_{0}^{\infty} \Gamma(x, y, t+n T) u_{0}(y) \mathrm{d} y\right| \leq \frac{C}{\sqrt{n T+t}}\left\|u_{0}\right\|_{L^{1}([0, \infty))}$ and thus

$\lim _{n \rightarrow \infty} \int_{0}^{\infty} \Gamma(x, y, n T+t) u_{0}(y) \mathrm{d} y=0$.

Denote the second integral in (2.30) by $u_{g}(x, n T+t)$ and consider the series

$u_{g}(x, t)+\sum_{k=1}^{\infty}\left[u_{g}(x,(k+1) T+t)-u_{g}(x, k T+t)\right]$

Since $g$ is periodic of period $T$,

$$
\begin{gathered}
u_{g}(x,(k+1) T+t)-u_{g}(x, k T+t) \\
=\int_{k T+t}^{(k+1) T+t} g(t-\tau) \Phi(x, \tau) \mathrm{d} \tau .
\end{gathered}
$$

Therefore, by the estimate of $\Phi$ in Theorem 2.3,

$$
\begin{aligned}
& \left|u_{g}(x,(k+1) T+t)-u_{g}(x, k T+t)\right| \\
& \quad \leq C \frac{T}{(k T+t)^{3 / 2}}\|g\|_{L^{\infty}} .
\end{aligned}
$$

Thus the series in (2.31) converges and consequently the limit

$\lim _{n \rightarrow \infty} u_{g}(x, n T+t)$

exists and we denote this limit by $u_{\infty}$. To show that $u_{\infty}$ is periodic of period $T$, we write:

$$
\begin{aligned}
& u_{\infty}(x, T+t)-u_{\infty}(x, t) \\
&= {\left[u_{\infty}(x, T+t)-u(x,(n+1) T+t)\right] } \\
&+[u(x,(n+1) T+t)-u(x, n T+t)] \\
&+\left[u(x, n T+t)-u_{\infty}(x, t)\right]
\end{aligned}
$$

and then let $n \rightarrow \infty$. Finally, the fact that $u(0, n T+t)=g(t)$ leads to the condition $u_{\infty}(0, t)=g(t)$. This completes the proof of Theorem 2.4.

\section{Numerical results}

In this section we present the results of our numerical experiments investigating the eventual periodicity of the full $\mathrm{KdV}$ equation and of the equations obtained by appending the $\mathrm{KdV}$ equation with two different types of dissipation.

More precisely, we compute the numerical solutions of the IBVP for the KdV equation:

$$
\begin{cases}u_{t}+u_{x}+u u_{x}+u_{x x x}=0, & x \geq 0, t \geq 0, \\ u(x, 0)=u_{0}(x), & x \geq 0, \\ u(0, t)=g(t), & t \geq 0,\end{cases}
$$

for suitable boundary data $g$. Since the initial data $u_{0}$ is not essential in determining the eventual periodicity, we simply set it to zero. We then plot the numerical solutions to examine their eventual periodicity. To understand how dissipation influences the eventual periodicity, we also compute the solutions of the IBVP for the KdV-Burgers equation:

$\begin{cases}u_{t}+u_{x}+u u_{x}+u_{x x x}-u_{x x}=0, & x \geq 0, t \geq 0, \\ u(x, 0)=u_{0}(x), & x \geq 0, \\ u(0, t)=g(t), & t \geq 0\end{cases}$ 

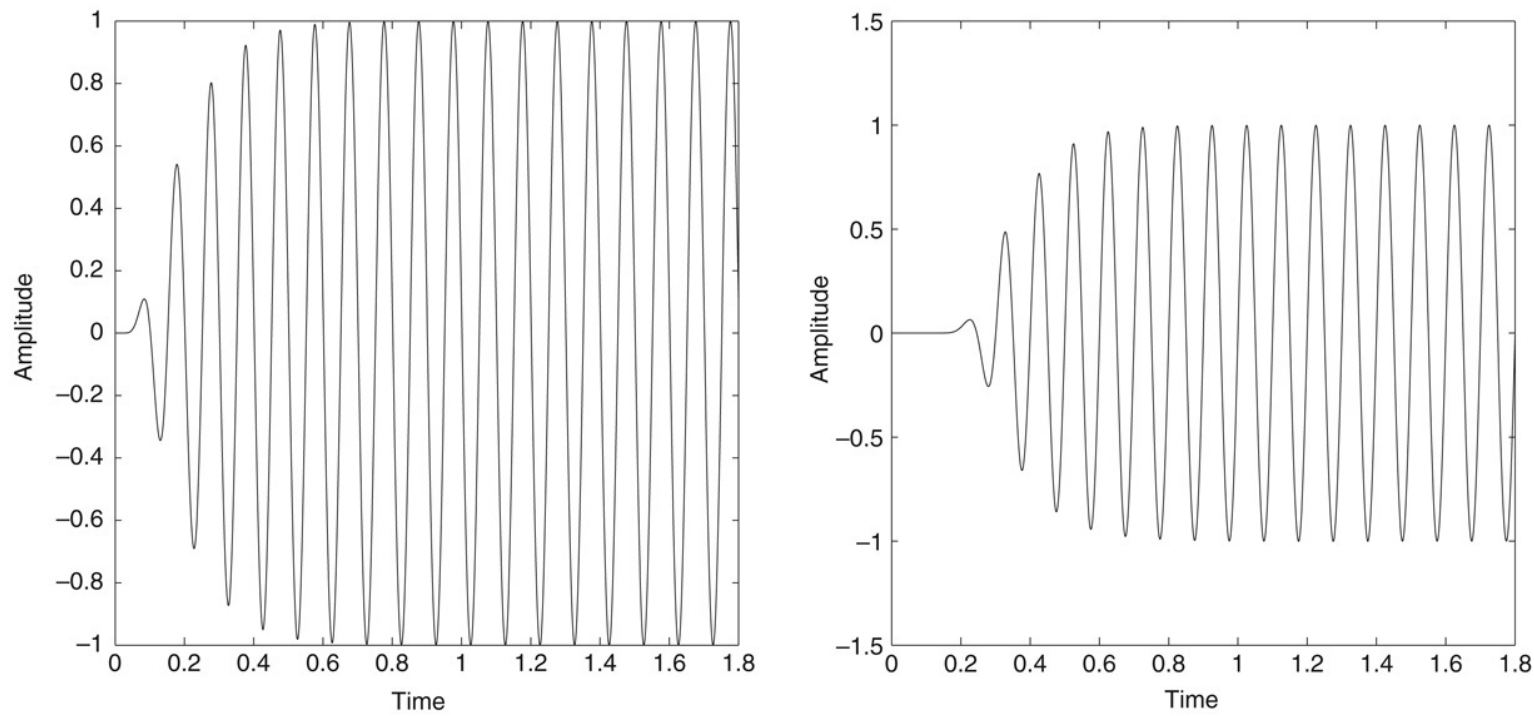

Fig. 1. $u(x, t)$ at $x=-0.95067$ (left graph) and at $x=-0.80846$ (right graph).
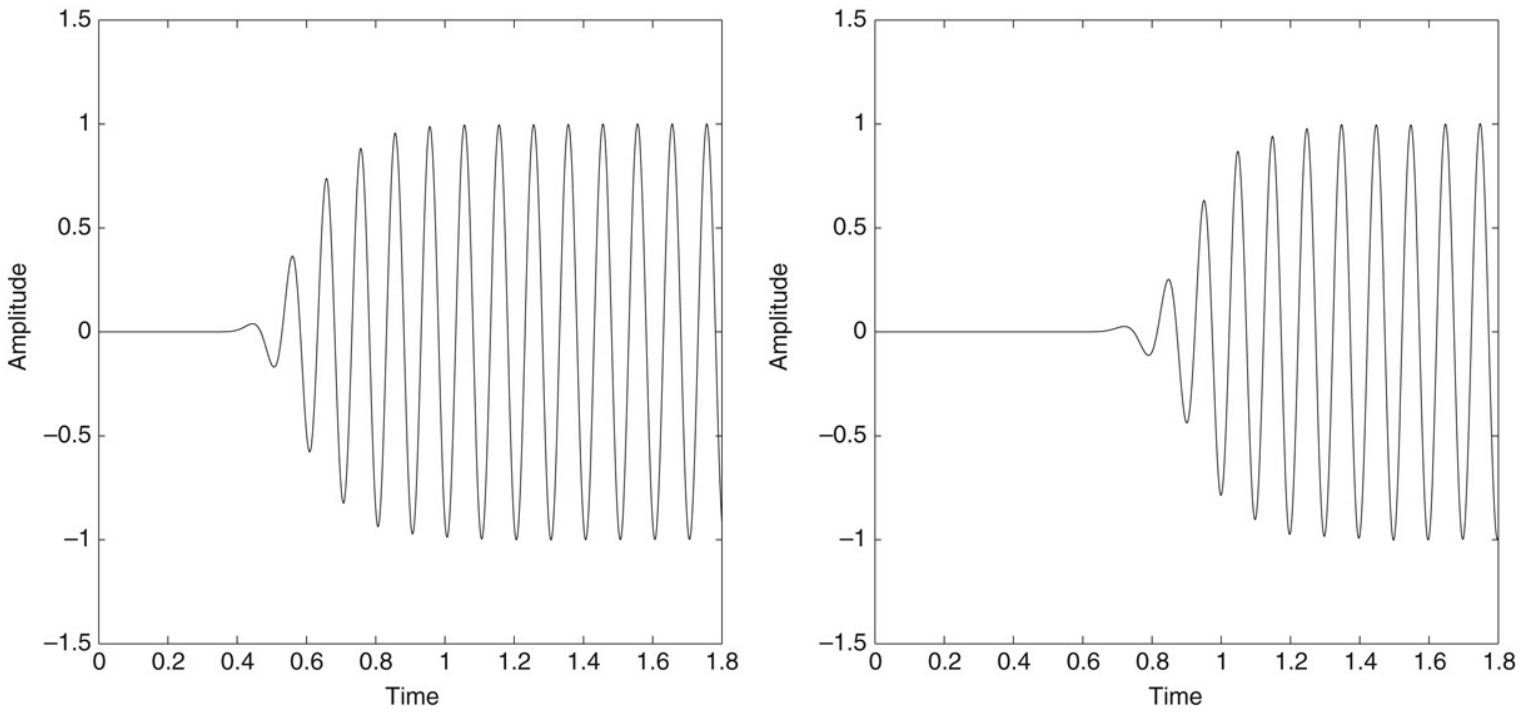

Fig. 2. $u(x, t)$ at $x=-0.58728$ (left graph) and at $x=-0.30872$ (right graph).

and the solutions to the following IBVP:

$$
\begin{cases}u_{t}+u+u_{x}+u u_{x}+u_{x x x}=0, & x \geq 0, t \geq 0, \\ u(x, 0)=u_{0}(x), & x \geq 0, \\ u(0, t)=g(t), & t \geq 0 .\end{cases}
$$

Since any solution $u(x, t)$ of these IBVPs converges to 0 as $x \rightarrow \infty$, we first approximate these IBVPs by the corresponding two-point boundary value problems for $x \in$ $[0, L]$ and then rescale the resulting problems to the interval $[-1,1]$. More precisely, we compute solutions of the following scaled problems:

$$
\begin{cases}u_{t}+\alpha u_{x}+\beta u u_{x}+\gamma u_{x x x}=0, & x \in[-1,1], t \in[0, T], \\ u(0, t)=g(t), & t \in[0, T] \\ u(1, t)=u_{x}(1, t)=0, & x \in[-1,1] \\ u(x, 0)=u_{0}(x), & \end{cases}
$$

$$
\begin{cases}u_{t}+\alpha u_{x}+\beta u u_{x} & \\ \quad+\gamma u_{x x x}-\delta u_{x x}=0, & x \in[-1,1], t \in[0, T], \\ u(0, t)=g(t), & \\ u(1, t)=u_{x}(1, t)=0, & t \in[0, T], \\ u(x, 0)=u_{0}(x), & x \in[-1,1],\end{cases}
$$

and

$$
\begin{cases}u_{t}+\eta u+\alpha u_{x} & \\ \quad+\beta u u_{x}+\gamma u_{x x x}=0, & x \in[-1,1], t \in[0, T], \\ u(0, t)=g(t), & \\ u(1, t)=u_{x}(1, t)=0, & t \in[0, T], \\ u(x, 0)=u_{0}(x), & x \in[-1,1],\end{cases}
$$

where $\alpha, \beta, \gamma, \delta$ and $\eta$ are parameters. For $u_{0} \equiv 0,(3.4)-(3.6)$ are valid approximations of (3.1)-(3.3), respectively, until the moment when the wave-front generated by the boundary data $g$ reaches the right boundary point $x=1$. 

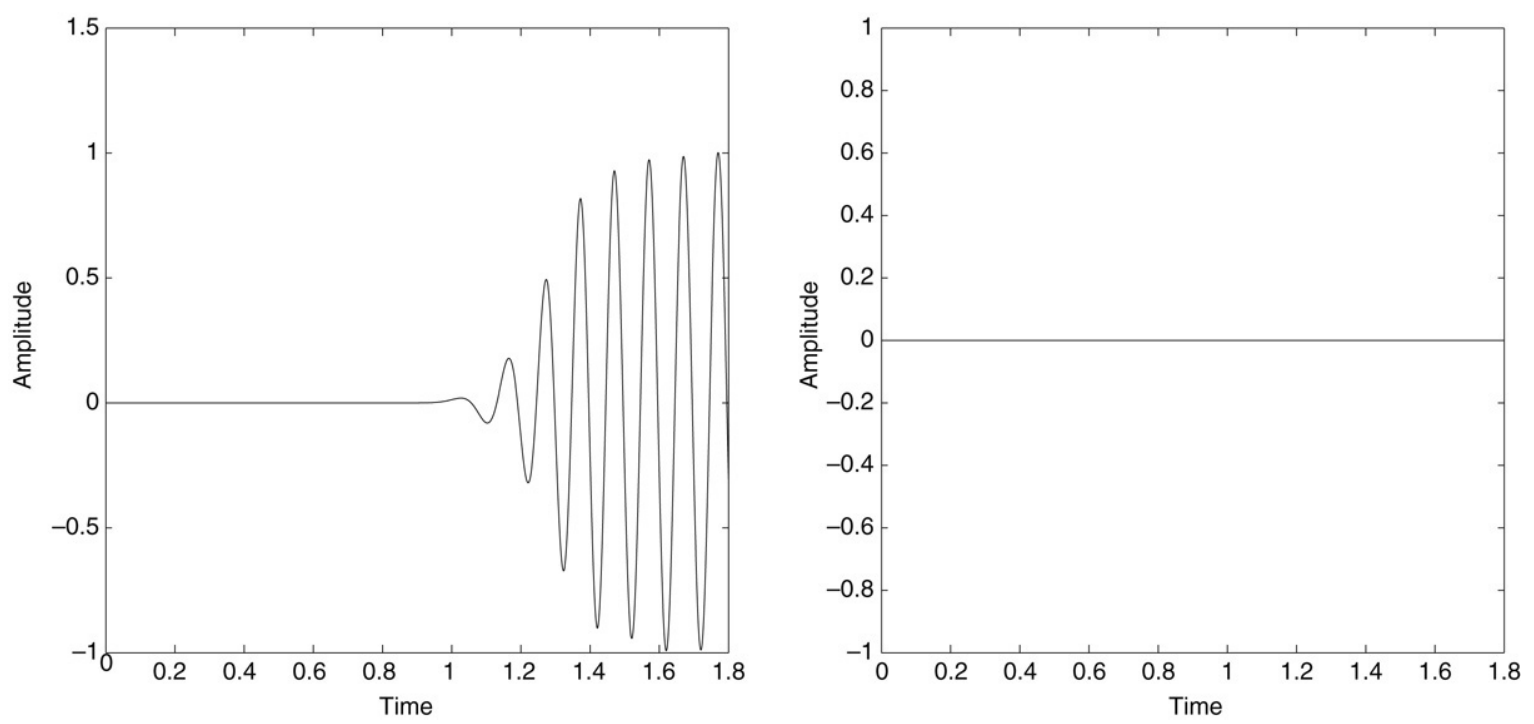

Fig. 3. $u(x, t)$ at $x=0$ (left graph) and at $x=0.99965$ (right graph).
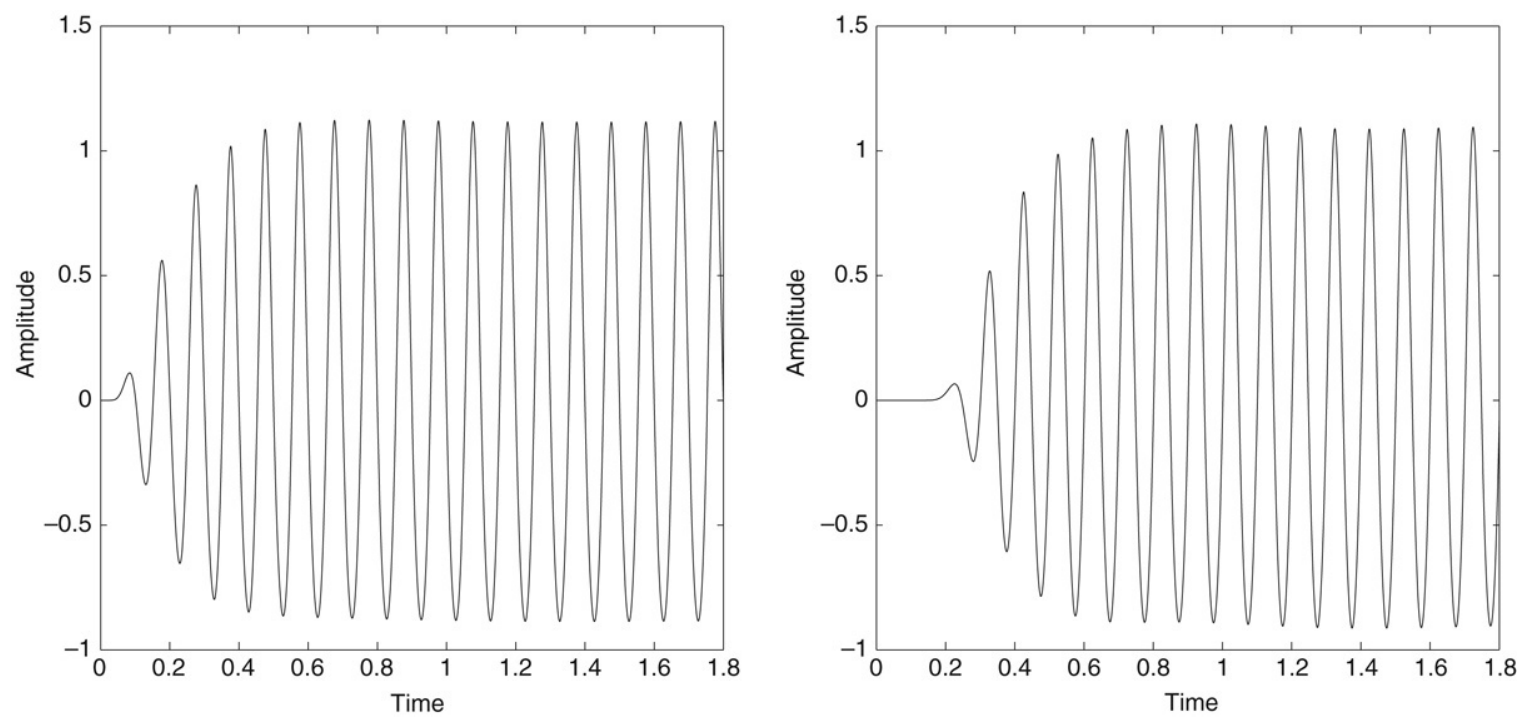

Fig. 4. $u(x, t)$ at $x=-0.95067$ (left graph) and at $x=-0.80846$ (right graph).

The solutions of (3.4)-(3.6) are computed using the Dual Petrov-Galerkin Method proposed by Shen in [8]. This method, suitable for third and higher odd-order equations, is very accurate and efficient. Detailed convergence estimates and numerical results exhibiting the accuracy and efficiency can be found in [8].

In our first set of experiments, we computed the solutions of the linearized KdV equation, namely (3.4) with $\beta=0$. Plotted here are the solutions of (3.4) with

$\alpha=1.0, \quad \gamma=10^{-5}$ and $\quad g(t)=\sin (20 \pi t) \tanh (5 t)$.

The six graphs in Figs. 1 through 3 record the amplitudes $u(x, t)$ for $x=-0.95067,-0.80846,-0.58728,-0.30872$, 0 and $x=0.99965$, and for $0<t<1.8$. In these graphs, the $x$ axis represents time $t$ and the $y$-axis represents the amplitude $u$.
The boundary data $g$ in (3.7) are not exactly periodic but become periodic. These graphs clearly show the eventual periodicity of the amplitudes at $x=-0.95067,-0.80846$, $-0.58728,-0.30872$ and 0 . The last plot in Fig. 3 shows that the amplitude at $x=0.99965$ remains zero. The purpose of this plot is to show that the wave front has not reached the right boundary for $t=1.8$ and thus the validity of the amplitudes at $x=-0.95067,-0.80846,-0.58728,-0.30872$ and 0 .

In the second set of experiments, we computed solutions of (3.4) with

$\alpha=1.0, \quad \beta=0.05, \quad \gamma=10^{-5} \quad$ and $g(t)=\sin (20 \pi t) \tanh (5 t)$.

We plot in Figs. 4 through 6 the amplitudes $u(x, t)$ corresponding to $x=-0.95067,-0.80846,-0.58728$, $-0.30872,0$ and $x=0.99965$, and for $0<t<1.8$. The 

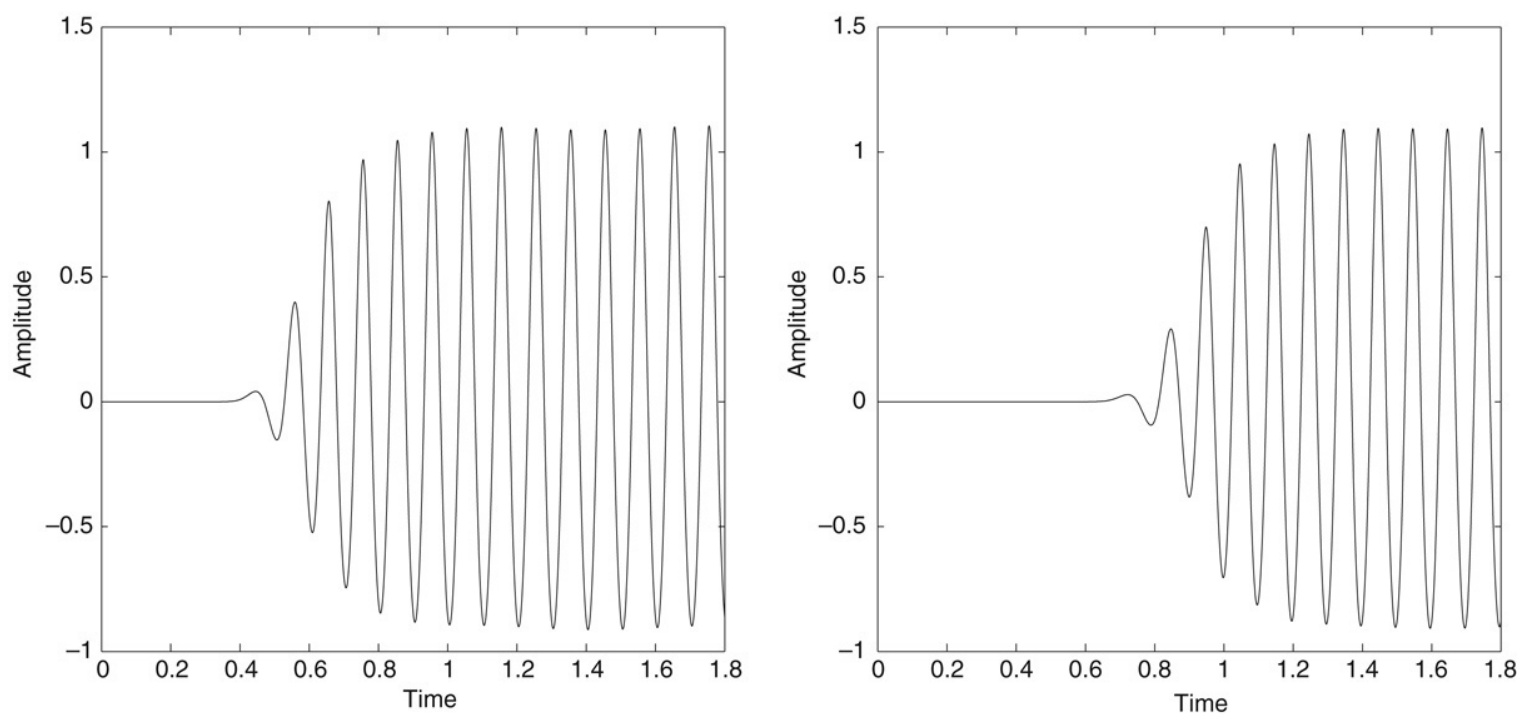

Fig. 5. $u(x, t)$ at $x=-0.58728$ (left graph) and at $x=-0.30872$ (right graph).
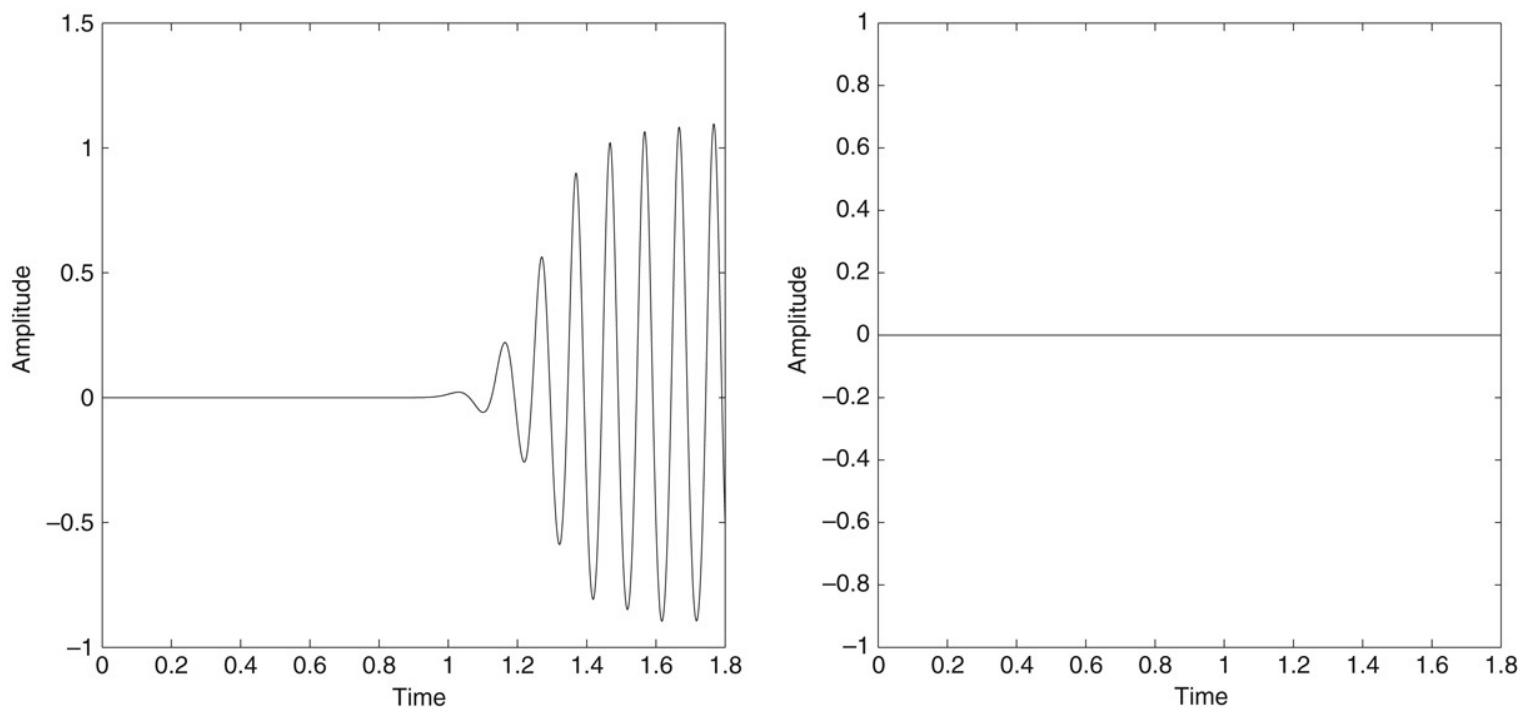

Fig. 6. $u(x, t)$ at $x=0$ (left graph) and at $x=0.99965$ (right graph).

graphs in these figures clearly indicate the eventual periodicity of the amplitudes at these positions. By comparing these plots with the ones for the linearized $\mathrm{KdV}$ equation, we also noticed the effects of the nonlinear term, which significantly "lifted" the amplitudes.

The third set of numerical experiments are performed on the IBVP for the KdV-Burgers equation (3.5). We recorded the results corresponding to

$\alpha=1.0, \quad \beta=0.05, \quad \gamma=10^{-5}$,

$\delta=10^{-4}$ and $g(t)=\sin (20 \pi t) \tanh (5 t)$.

in Figs. 7 through 9. Again we observe the pattern of eventual periodicity in $u(x, t)$ at all selected positions. The effects of the Burgers type dissipation are reflected in the damped amplitude.

We have also computed the solutions of the IBVP (3.6) in order to understand the effects of the dissipative term $\eta u$ on the eventual periodicity for a general boundary data. We plot in Figs. 10 through 12 the solutions of (3.6) corresponding to

$\eta=5, \quad \alpha=1, \quad \beta=0.05, \quad \gamma=10^{-4} \quad$ and

$g(t)=\sin (20 \pi t) \tanh (5 t)$

and at the positions $x=-0.95067,-0.80846,-0.58728$, $-0.30872,0$ and $x=0.58728$. We notice that the amplitudes are significantly damped by the dissipation but the pattern of eventual periodicity is still maintained.

\section{Acknowledgements}

J. Yuan is partially supported by the Mathematics Department of Oklahoma State University and the National Science Council of the Republic of China under the grant NSC 94-2115-M-126-004and 95-2115-M-126-003. The work of J. Shen is supported in part by NSF DMS-0610646. 

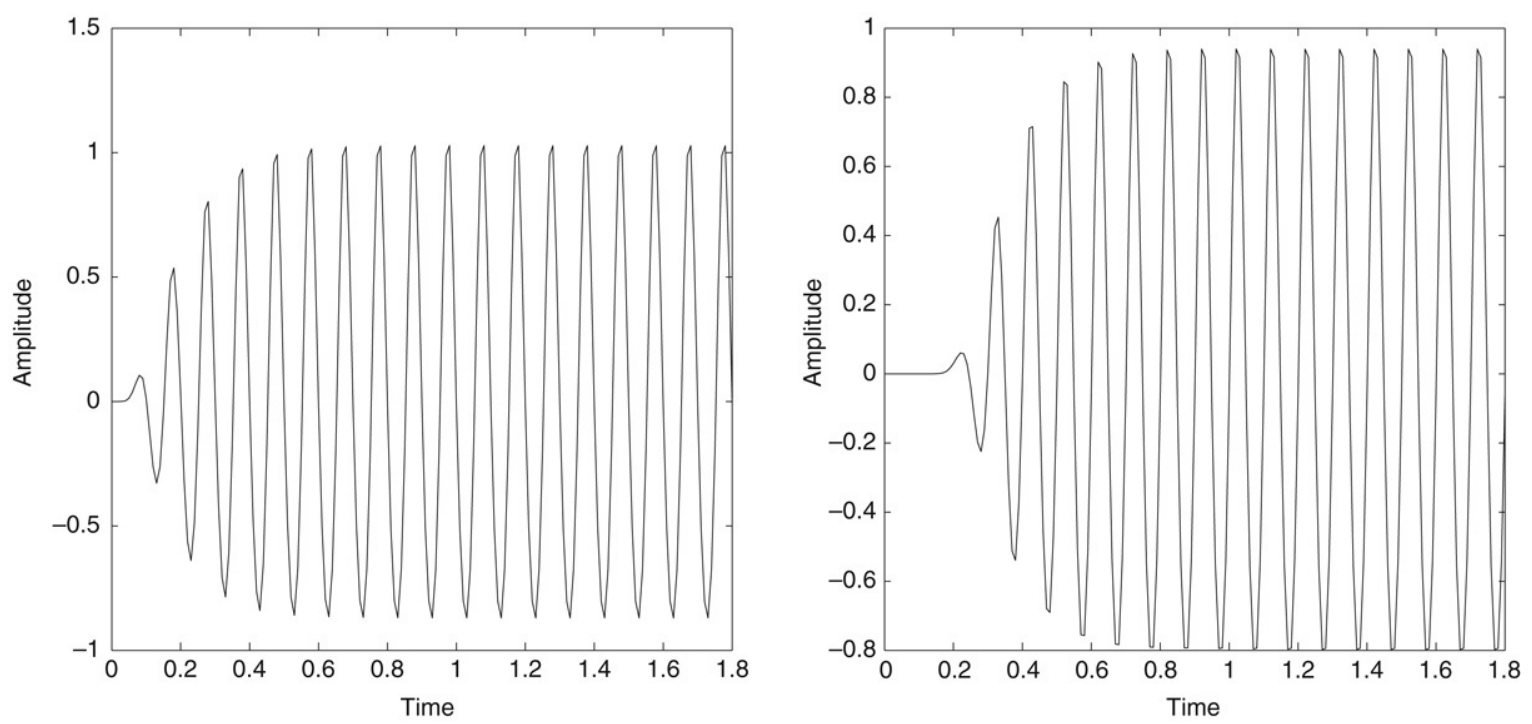

Fig. 7. $u(x, t)$ at $x=-0.95067$ (left graph) and at $x=-0.80846$ (right graph).
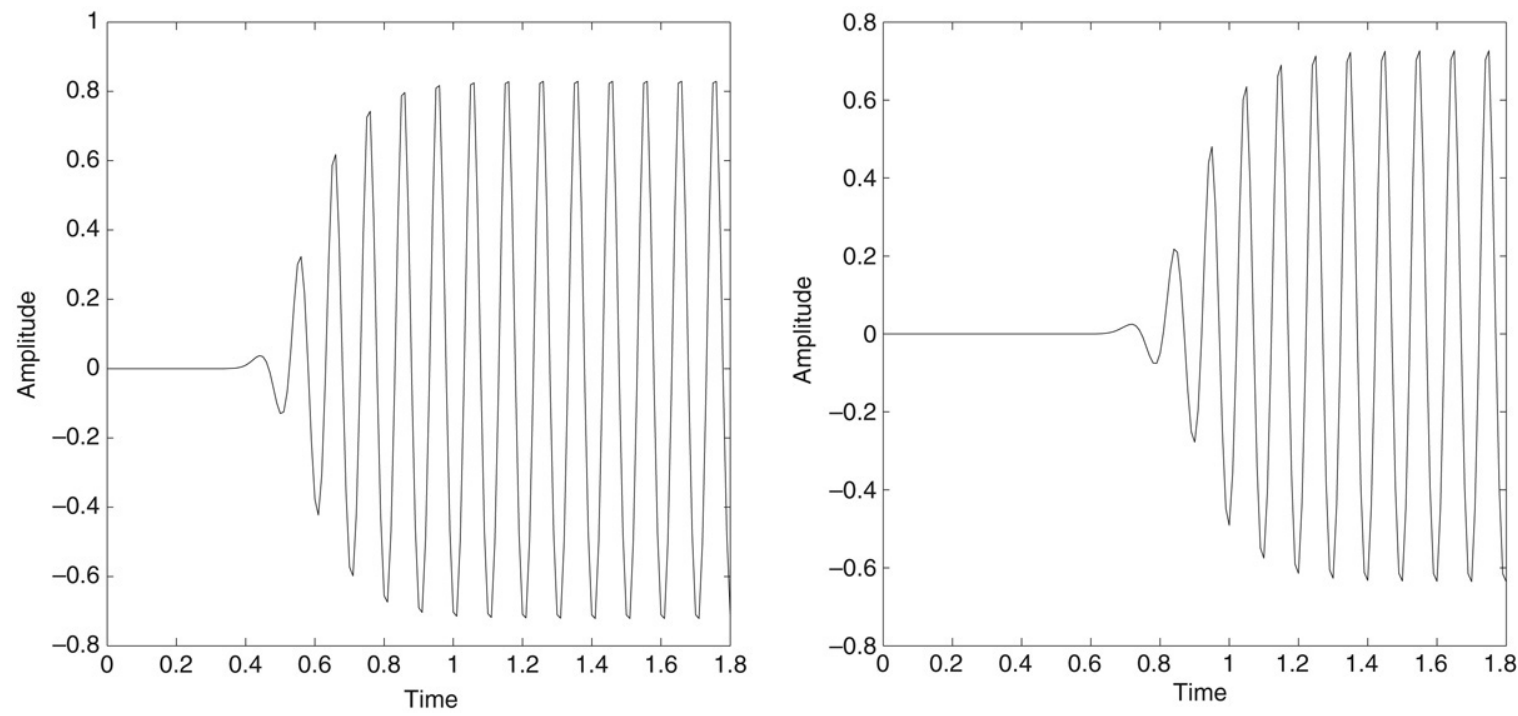

Fig. 8. $u(x, t)$ at $x=-0.58728$ (left graph) and at $x=-0.30872$ (right graph).

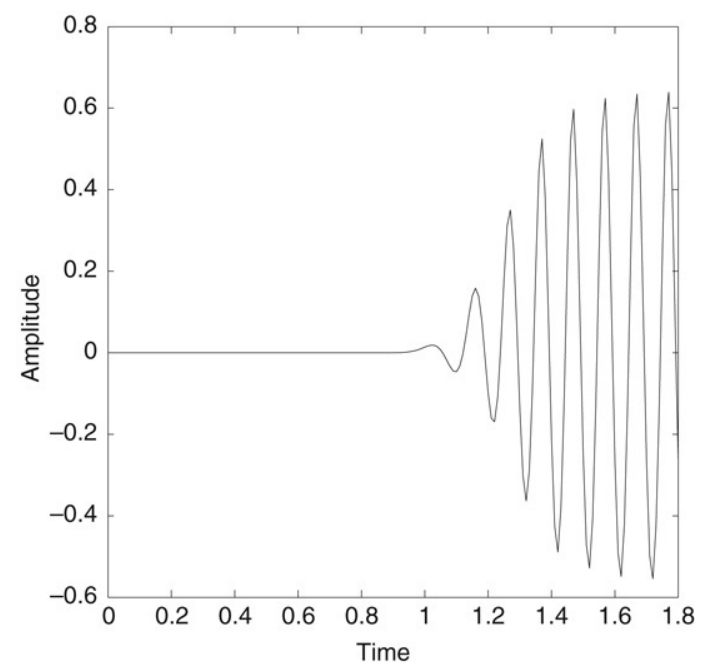

Fig. 9. $u(x, t)$ at $x=0$.
J. Shen and J. Wu thank the National Centre for Theoretical Sciences at Taipei and the Mathematics Research Promotion Centre, Taiwan, for support and hospitality during part of this collaboration. We also thank Professors J. Bona and M. Chen for valuable discussions and the referees for helpful comments.

\section{Appendix A}

This appendix provides several elementary properties of the Laplace and inverse Laplace transforms.

For a real- or complex-valued function $F$ of $x \geq 0$, its Laplace transform $\widehat{F}$ is defined by (2.2), namely

$(\mathcal{L} F)(\xi)=\widehat{F}(\xi)=\int_{0}^{\infty} \mathrm{e}^{-\xi x} F(x) \mathrm{d} x$.

The first property concerns the convergence of this integral. We recall that a function $F$ is said to be of exponential order $\alpha$ if 

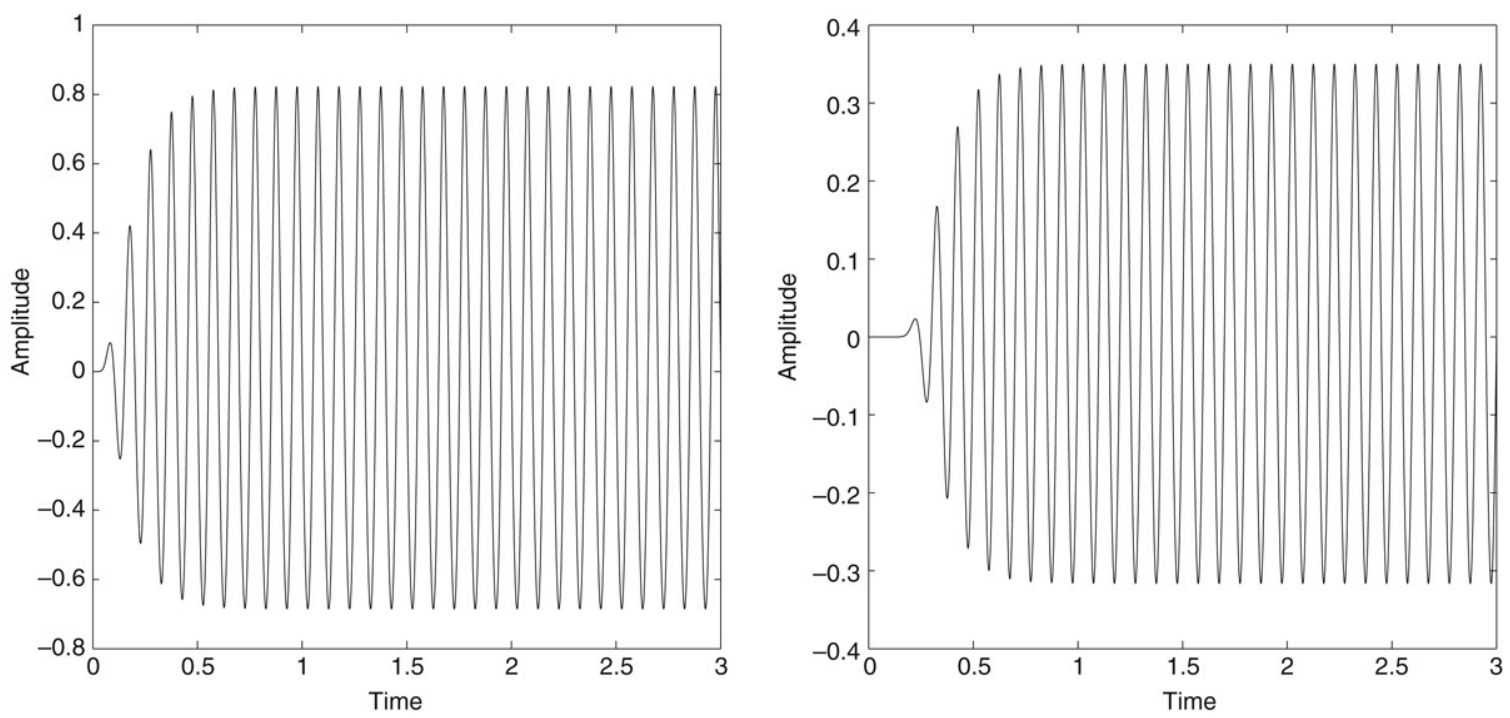

Fig. 10. $u(x, t)$ at $x=-0.95067$ (left graph) and at $x=-0.80846$ (right graph).
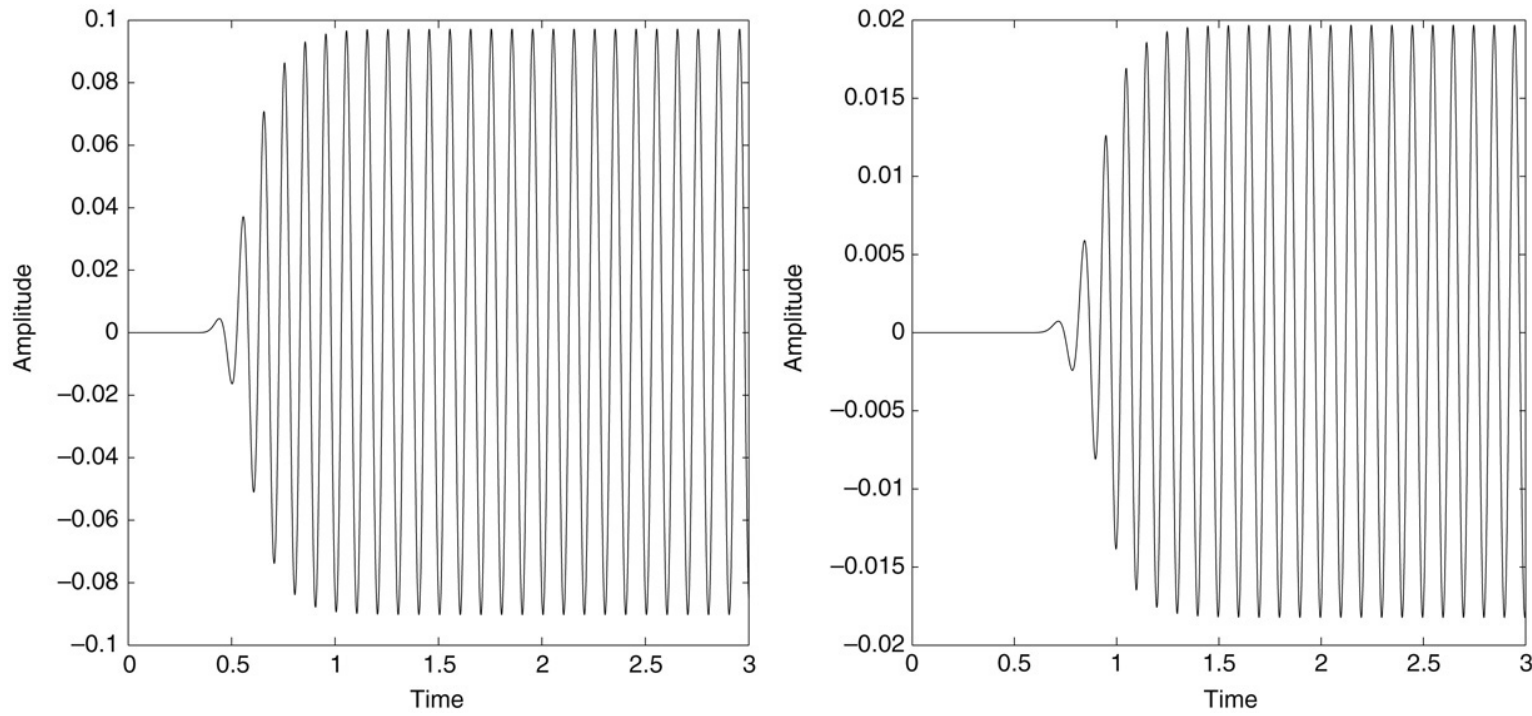

Fig. 11. $u(x, t)$ at $x=-0.58728$ (left graph) and at $x=-0.30872$ (right graph).

there exist constants $M>0$ and $\alpha$ such that for some $x_{0} \geq 0$,

$|F(x)| \leq M \mathrm{e}^{\alpha x}, \quad x \geq x_{0}$.

Proposition A.1. Assume that $F$ is piecewise continuous on $[0, \infty)$ and of exponential order $\alpha$. Then:

(1) $\mathcal{L} F$ defined in (2.2) exists and converges absolutely for $\operatorname{Re}(\xi)>\alpha$. In addition, the convergence is uniform for $\operatorname{Re}(\xi) \geq x_{0}>\alpha$

(2) $\widehat{F}(\xi) \rightarrow 0$ as $\operatorname{Re}(\xi) \rightarrow \infty$.

Proposition A.2. Let $n \geq 0$ be an integer. Suppose that $F$, $F^{\prime}, \ldots, F^{(n-1)}$ are continuous on $[0, \infty)$ and of exponential order, while $F^{(n)}$ is piecewise continuous on $[0, \infty)$. Then:

$$
\begin{aligned}
\left(\mathcal{L} F^{(n)}\right)(\xi)= & \xi^{n}(\mathcal{L} F)(\xi)-\xi^{n-1} F\left(0^{+}\right) \\
& -\xi^{n-2} F^{\prime}\left(0^{+}\right)-\cdots-F^{(n-1)}\left(0^{+}\right) .
\end{aligned}
$$

The inverse Laplace transform defined in (2.3) maps the Laplace transform of a function back to the original function. The following proposition states necessary and sufficient conditions for a given function to have a convergent inverse Laplace transform. This result can be found in [4].

Proposition A.3. The necessary and sufficient conditions for a given function $H(\xi)$ to have an inverse Laplace transform $h(x)$ that is continuous and of exponential order $\alpha$ are:

(a) $H(\xi)$ is analytic for $\operatorname{Re}(\xi)>\alpha$;

(b) $\|H(b+\mathrm{i} \cdot)\|_{L^{1}(-\infty, \infty)}<\infty$ for any $b>\alpha$;

(c) For any $\epsilon>0$ and $b_{0}>\alpha$, there exist $M>0$ and $m>0$ such that

$|H(\xi)| \leq M \mathrm{e}^{\epsilon b}\left(1+|\xi|^{m}\right), \quad b>b_{0}$. 

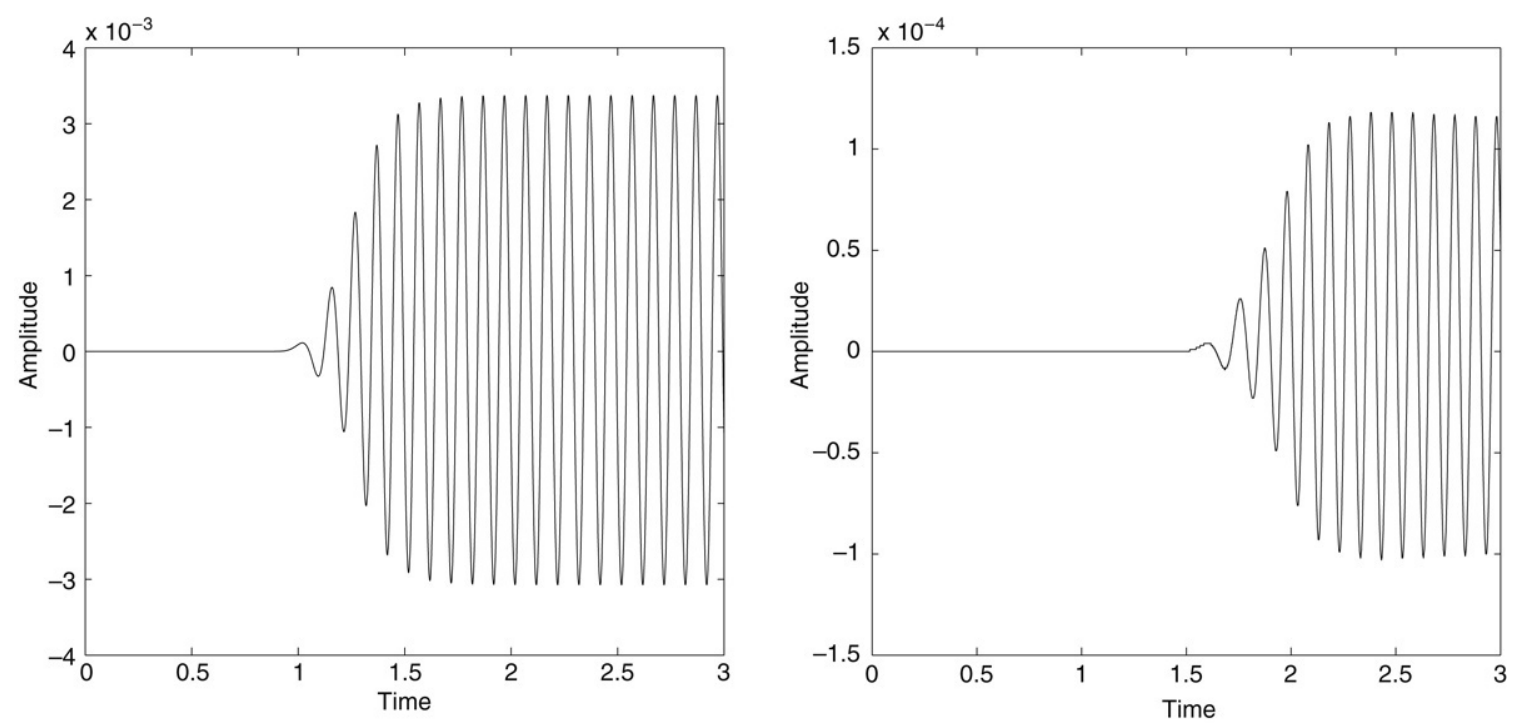

Fig. 12. $u(x, t)$ at $x=0$ (left graph) and at $x=0.58728$ (right graph).

When these conditions are satisfied, $h(x)$ can be computed by:

$h(x)=\frac{1}{2 \pi \mathrm{i}} \int_{b-\mathrm{i} \infty}^{b+\mathrm{i} \infty} \mathrm{e}^{\xi x} H(\xi) \mathrm{d} \xi$

for any $b>\alpha$.

\section{Appendix B}

This appendix offers an expanded commentary on the asymptotic analysis of the various oscillatory integrals arising in Section 2.2. The asymptotic analysis relies upon standard results in the theory of stationary phase, e.q. Theorem 13.1 in Olver's book [6]. For readers' convenience, we recall this theory here.

Suppose that in the integral

$I(t)=\int_{a}^{b} \mathrm{e}^{\mathrm{i} t p(y)} q(y) \mathrm{d} y$

the limits $a$ and $b$ are independent of $t, a$ being finite and $b(>a)$ finite or infinite. The functions $p(y)$ and $q(y)$ are independent of $t, p(y)$ being real and $q(y)$ either real or complex. We also assume that the only point at which $p^{\prime}(y)$ vanishes is $a$. Without loss of generality, both $t$ and $p^{\prime}(y)$ are taken to be positive; cases in which one of them is negative can be handled by changing the sign of $i$ throughout. We require:

(i) In $(a, b)$, the functions $p^{\prime}(y)$ and $q(y)$ are continuous, $p^{\prime}(y)>0$, and $p^{\prime \prime}(y)$ and $q^{\prime}(y)$ have at most a finite number of discontinuities and infinities:

(ii) As $y \rightarrow a+$,

$$
p(y)-p(a) \sim P(y-a)^{\mu}, \quad q(y) \sim Q(y-a)^{\lambda-1},
$$

the first of these relations being differentiable. Here $P, \mu$ and $\lambda$ are positive constants, and $Q$ is a real or complex constant; (iii) For each $\epsilon \in(0, b-a)$,

$$
\mathcal{V}_{a+\epsilon, b}\left\{\frac{q(y)}{p^{\prime}(y)}\right\} \equiv \int_{a+\epsilon}^{b}\left|\left(\frac{q(y)}{p^{\prime}(y)}\right)^{\prime}\right| \mathrm{d} y<\infty ;
$$

(iv) As $t \rightarrow b-$, the limit of $q(y) / p^{\prime}(y)$ is finite, and this limit is zero if $p(b)=\infty$.

With these conditions, the nature of asymptotic approximation to $I(t)$ for large $t$ depends on the sign of $\lambda-\mu$. In the case $\lambda<\mu$, we have the following theorem:

Theorem B.1. In addition to the above conditions, assume that $\lambda<\mu$, the first of (B.1) is twice differentiable, and the second of (B.1) is differentiable, then

$I(t) \sim \mathrm{e}^{\lambda \pi \mathrm{i} /(2 \mu)} \frac{Q}{\mu} \Gamma\left(\frac{\lambda}{\mu}\right) \frac{\mathrm{e}^{\mathrm{i} t p(a)}}{(P t)^{\lambda / \mu}}$

as $t \rightarrow \infty$.

We now provide the details leading to (2.27). It suffices to check the conditions of Theorem B.1. Setting

$a=-\frac{1}{\sqrt{3}}, \quad b=0, \quad p(\sigma)=\sigma-\sigma^{3} \quad$ and
$q(\sigma)=\mathrm{e}^{-\mathrm{i} \sigma x}$,

we have

(i) $p, p^{\prime}, p^{\prime \prime}, q$ and $q^{\prime}$ are all continuous in $(-1 / \sqrt{3}, 0)$, and $p^{\prime}(\sigma)>0$.

(ii) As $\sigma \rightarrow-\frac{1}{\sqrt{3}}+$,

$p(\sigma)-p\left(-\frac{1}{\sqrt{3}}\right) \sim \sqrt{3}\left(\sigma+\frac{1}{\sqrt{3}}\right)^{2}$,

$q(\sigma) \sim \mathrm{e}^{\mathrm{i} \frac{1}{\sqrt{3}} x}$

That is, $P=\sqrt{3}, \mu=2, Q=\mathrm{e}^{\mathrm{i} \frac{1}{\sqrt{3}} x}$ and $\lambda=1$. 
(iii) For any fixed $\epsilon>0, \mathcal{V}_{-\frac{1}{\sqrt{3}}+\epsilon, 0}\left(q / p^{\prime}\right)<\infty$. In fact,

$$
\begin{gathered}
\mathcal{V}_{-\frac{1}{\sqrt{3}}+\epsilon, 0}\left(\frac{q}{p^{\prime}}\right)=\int_{-\frac{1}{\sqrt{3}}+\epsilon}^{0}\left|\left(\frac{q(\sigma)}{p^{\prime}(\sigma)}\right)^{\prime}\right| \mathrm{d} \sigma \\
=\int_{-\frac{1}{\sqrt{3}}+\epsilon}^{0}\left|\frac{\mathrm{i} x\left(3 \sigma^{2}-1\right)+6 \sigma}{\left(1-3 \sigma^{2}\right)^{2}}\right| \mathrm{d} \sigma<\infty .
\end{gathered}
$$

(iv) As $\sigma \rightarrow 0-, q / p^{\prime}=\mathrm{e}^{-\mathrm{i} \sigma x} /\left(1-3 \sigma^{2}\right) \rightarrow 1$.

Theorem B.1 then implies:

$$
\begin{aligned}
\int_{-\frac{1}{\sqrt{3}}}^{0} \mathrm{e}^{\mathrm{i}\left(\sigma-\sigma^{3}\right) t} \mathrm{e}^{-\mathrm{i} \sigma(x-y)} \mathrm{d} \sigma & \sim \mathrm{e}^{\frac{\pi \mathrm{i}}{4}} \frac{1}{2} \mathrm{e}^{\mathrm{i} \frac{x-y}{\sqrt{3}}} \Gamma\left(\frac{1}{2}\right) \frac{\mathrm{e}^{\mathrm{i} t\left(-\frac{2}{3 \sqrt{3}}\right)}}{(\sqrt{3} t)^{1 / 2}} \\
& =\frac{\sqrt{\pi} \mathrm{e}^{\frac{\pi \mathrm{i}}{4}} \mathrm{e}^{\frac{\mathrm{i}}{\sqrt{3}}\left(x-y-\frac{2}{3} t\right)}}{2 \sqrt[4]{3} \sqrt{t}} .
\end{aligned}
$$

The estimate (2.28) also follows from Theorem B.1. The conditions can be similarly checked for this integral. In fact, for

$a=\frac{1}{\sqrt{3}}, \quad b=\infty, \quad p(\sigma)=\sigma^{3}-\sigma \quad$ and $q(\sigma)=\mathrm{e}^{\mathrm{i} \sigma x}$,

we have

$p(\sigma)-p\left(\frac{1}{\sqrt{3}}\right) \sim \sqrt{3}\left(\sigma-\frac{1}{\sqrt{3}}\right)^{2} \quad$ and $\quad q(\sigma) \sim \mathrm{e}^{\mathrm{i} \frac{1}{\sqrt{3}} x}$ as $\sigma \rightarrow \frac{1}{\sqrt{3}}+$. Other conditions can also be verified. Theorem B.1 then says

$$
\begin{aligned}
\int_{\frac{1}{\sqrt{3}}}^{\infty} \mathrm{e}^{\mathrm{i}\left(\sigma^{3}-\sigma\right) t} \mathrm{e}^{\mathrm{i} \sigma(x-y)} \mathrm{d} \sigma & \sim \mathrm{e}^{\frac{\pi \mathrm{i}}{4}} \frac{1}{2} \mathrm{e}^{\mathrm{i} \frac{x-y}{\sqrt{3}}} \Gamma\left(\frac{1}{2}\right) \frac{\mathrm{e}^{\mathrm{i} t\left(-\frac{2}{3 \sqrt{3}}\right)}}{(\sqrt{3} t)^{1 / 2}} \\
& =\frac{\sqrt{\pi} \mathrm{e}^{\frac{\pi \mathrm{i}}{4}} \mathrm{e}^{\frac{\mathrm{i}}{\sqrt{3}}\left(x-y-\frac{2}{3} t\right)}}{2 \sqrt[4]{3} \sqrt{t}} .
\end{aligned}
$$

\section{References}

[1] J.L. Bona, W.G. Pritchard, L.R. Scott, An evaluation of a model equation for water waves, Philos. Trans. R. Soc. Lond. Ser. A 302 (1981) 457-510.

[2] J.L. Bona, S.M. Sun, B.-Y. Zhang, Forced Oscillations of a damped Korteweg-de Vries equation in a quarter plane, Comm. Contemp. Math. 5 (2003) 369-400.

[3] J.L. Bona, J. Wu, Temporal growth and eventual periodicity for dispersive wave equations in a quarter plane. Discrete Contin. Dyn. Syst. (in press).

[4] N. Hayashi, E.I. Kaikina, Nonlinear Theory of Pseudodifferential Equations on a Half-Line, in: North-Holland Mathematics Studies, vol. 194, Elsevier Science B.V., Amsterdam, 2004.

[5] N. Hayashi, E.I. Kaikina, J.L. Guardado Zavala, On the boundary-value problem for the Korteweg-de Vries equation, Proc. R. Soc. Lond. A. 459 (2003) 2861-2882.

[6] F. Olver, Asymptotics and Special Functions, Academic Press, New York, London, 1974.

[7] J. Schiff, The Laplace Transform, Springer-Verlag, New York, 1999.

[8] J. Shen, A new dual-Petrov-Galerkin method for third and higher odd-order differential equations: Application to the KdV euqation, SIAM J. Numer. Anal. 41 (2003) 1595-1619. 